\title{
CHI3L1 regulates PD-L1 and anti-CHI3L1-PD-1 antibody elicits synergistic antitumor responses
}

\author{
Bing Ma, ${ }^{1}$ Bedia Akosman, ${ }^{1}$ Suchitra Kamle, ${ }^{1}$ Chang-Min Lee, ${ }^{1}$ Chuan Hua He, ${ }^{1}$ Ja Seok Koo, ${ }^{2}$ Chun Geun Lee, ${ }^{1}$ and Jack A. Elias ${ }^{1,3}$ \\ 'Department of Molecular Microbiology and Immunology, Brown University, Providence, Rhode Island, USA. ${ }^{2}$ Section of Medical Oncology, Department of Internal Medicine, Yale Cancer Center, Yale School of \\ Medicine, New Haven, Connecticut, USA. ${ }^{3}$ Department of Medicine, Brown University, Providence, Rhode Island, USA.
}

\begin{abstract}
Evasion of the immune response is a hallmark of cancer, and programmed cell death 1 (PD-1) and PD-1 ligand 1 (PD-L1) are major mediators of this immunosuppression. Chitinase 3-like 1 (CHI3L1) is induced in many cancers, where it portends a poor prognosis and contributes to tumor metastasis and spread. However, the mechanism(s) that CHI3L1 uses in metastasis have not been defined. Here we demonstrate that CHI3L1 regulates the expression of PD-L1, PD-L2, PD-1, LAC3, and TIM3 and plays a critical role in melanoma progression and lymphatic spread. CHI3L1 also contributed to IFN- $\gamma-$ stimulated macrophage PD-L1 expression, and RIG-like helicase innate immunity suppressed CHI3L1, PD-L1, and melanoma progression. Individual antibodies against CHI3L1 or PD-1 had discrete antitumor effects and additive antitumor responses in metastasis models and T cell-tumor cell cocultures when administered simultaneously. Synergistic cytotoxic tumor cell death was seen in T cell-tumor cell cocultures, and significantly enhanced antitumor responses were seen in in vivo tumor models treated with bispecific antibodies that simultaneously target CHI3L1 and PD-1. CHI3L1 contributes to tumor progression by stimulating the PD-1/PD-L1 axis and other checkpoint molecules. The simultaneous targeting of CHI3L1 and the PD-1/PD-L1 axis with individual and, more powerfully, with bispecific antibodies represents a promising therapy for pulmonary metastasis and progression.
\end{abstract}

\section{Introduction}

One of the most exciting recent discoveries in cancer is the appreciation that evasion of the immune system and suppression of neoantigen-induced $\mathrm{T}$ cell responses are essential for cancer development, progression, and resistance to treatment (1-3). Studies of these responses have highlighted essential immune checkpoints (ICPs) that are regulated by inhibitory receptors and their ligands such as programed death-1 (PD-1) and its ligands PD-1 ligand 1 (PD-L1) and PD-L2; cytotoxic T lymphocyte-associated antigen 4 (CTLA4) and its ligands B7.1 and B7.2; and lymphocyte activation gene 3 protein (LAG3) and its ligand HLA class II $(4,5)$. These advances have led to the development of ICP molecule-blocking antibodies against PD-1, PD-L1, and CTLA4, which have proven to be impressively successful therapeutics for a number of malignancies $(2,4)$. This is particularly important in the lung, because recent studies demonstrated that ICP antibodies can be useful therapeutics in lung cancer (6-8). In these and other studies of ICP inhibitor-based therapeutics, some patients respond impressively while others do not. In many cases responsiveness to interventions in the PD-1/PD-L1 axis correlates with the expression of

Authorship note: BM, BA, and SK contributed equally to this work.

Conflict of interest: JAE is a cofounder of Elkurt Therapeutics and is a founder of and stockholder in and serves on the Scientific Advisory Board for Ocean Biomedical Inc., which develops inhibitors of 18-glycosyl hydrolases as therapeutics. JAE, CGL, and SK have a patent entitled "Methods and composition relating to anti-CHI3L1 antibody reagents" (US Patent 10253111).

Copyright: @ 2021, American Society for Clinical Investigation.

Submitted: March 2, 2020; Accepted: September 2, 2021; Published: November 1, 2021. Reference information: / Clin Invest. 2021;131(21):e137750

https://doi.org/10.1172/JCI137750. molecules like PD-L1 (6-8). However, the moieties that regulate the expression of ICP molecules and the complex mechanisms that they employ (9) have not been adequately identified.

The glycosyl hydrolase family 18 (GH18) proteins are members of an ancient gene family that exists in species as diverse as plants, insects, and humans $(10,11)$. This gene family contains true chitinases, which degrade chitin polysaccharides, and chitinase-like proteins (CLPs), which bind to but do not degrade chitin. These GH18 moieties have evolved during speciation with an impressive increase in CLPs coinciding with the appearance of mammals (10). This retention over species and evolutionary time has led to the belief that these moieties play essential roles in biologic homeostasis. However, their roles in biology have only recently begun to be defined. Chitinase 3-like 1 (CHI3L1; also called YKL40), the prototypic CLP, was originally discovered in mouse breast cancer cells (12). It is now known to be expressed by a variety of cells, including macrophages, neutrophils, and epithelial cells, and is stimulated by a number of mediators, including IL-13, IL-6, IL-1 $\beta$, and IFN- $\gamma(13-16)$. Studies from our laboratory and others have also demonstrated that CHI3L1 is a multifaceted moiety that inhibits cell death (apoptosis and pyroptosis), stimulates Th2 inflammation and M2 macrophage differentiation, inhibits oxidant injury, controls inflammasome and caspase activation, regulates TGF- $\beta 1$ elaboration, contributes to antibacterial responses, and activates MAP kinase (MAPK), Akt/protein kinase B, and Wnt/ $\beta$-catenin signaling $(13,17-20)$. They also demonstrated that many of these responses are mediated by a multimeric receptor called the chitosome that contains an IL-13 receptor $\alpha 2$ (IL-13R $\alpha 2$ ) subunit and a TMEM219 $\beta$ subunit $(18,21)$. In keeping with these diverse sources and stimuli, elevated levels of CHI3L1 have been 
noted in a wide variety of diseases characterized by inflammation, fibrosis, and tissue remodeling (14, 22-25). However, the roles of CHI3L1 in these diverse diseases have not been fully defined.

Recent studies demonstrated that the levels of circulating CHI3L1 are increased in many malignancies, including cancers of the prostate, colon, rectum, ovary, kidney, and breast as well as glioblastomas and malignant melanoma (26-38). In these diseases, the levels of CHI3L1 frequently correlate directly with disease progression and inversely with disease-free interval and survival (26-38). This is particularly striking in lung cancer, in which the serum and tissue levels of CHI3L1 are impressively increased and correlate with adverse outcomes (27, 39-41). CHI3L1 may also play a particularly important role in pulmonary metastasis and tumor progression; studies from our laboratory and others have demonstrated that CHI3L1 induction is required for the generation of a metastasis-permissive pulmonary microenvironment (42), and metastatic spread can be inhibited via RIG-like helicase (RLH) innate immune inhibition of CHI3L1 elaboration (43). However, the mechanism(s) by which CHI3L1 contributes to tumor initiation and spread have not been adequately defined, and the degree to which CHI3L1 mediates its tumorigenic effects via activation of ICP pathways such as the PD-1/PD-L1 axis has not been addressed. In addition, although recent studies reported that RLH activation is critical for responsiveness to ICP blockade (44), the possibility that this responsiveness is mediated by RLH immune inhibition of CHI3L1 has not been considered.

We hypothesized that CHI3L1 contributes to pulmonary metastasis and spread via the regulation of ICP molecules. To address this hypothesis, studies were undertaken to determine whether CHI3L1 regulates the expression of components of the PD-1/PD-L1 axis, LAG3, or TIM3. In vivo studies demonstrated that PD-L1, PD-L2, PD-1, LAG3, and TIM3 are induced by melanoma metastasis and spread via CHI3L1-dependent mechanisms and that transgenic CHI3L1 stimulates these checkpoint inhibitors. In vitro studies demonstrated that CHI3L1 stimulates macrophage PD-L1 via a mechanism(s) that uses IL-13R $\alpha 2$ and that optimal IFN- $\gamma$ stimulation of macrophage PD-L1 requires CHI3L1. Our studies also demonstrated that RLH innate immune activation suppressed CHI3L1, PD-L1, and pulmonary metastasis. From a therapeutic perspective, antibodies against CHI3L1 or PD-1 had discrete antitumor effects, and at least additive antitumor effects when administered simultaneously, in metastasis models. Synergistic cytotoxic T lymphocyte-induced (CTL-induced) tumor cell death was seen in T cell-tumor cocultures, and significantly enhanced antitumor responses were seen in in vivo tumor models when treated with bispecific antibodies that simultaneously target CHI3L1 and PD-1. These studies demonstrate that CHI3L1 contributes to the development and/or progression of metastatic tumors in the lung via regulation of the PD-1/PD-L1 axis and other ICP molecules. They also strongly support the concept that the simultaneous inhibition of CHI3L1 and components of the PD-1/PD-L1 axis with monospecific antibodies and, even more powerfully, with bispecific antibodies represents an attractive therapeutic strategy for melanoma progression and spread.

\section{Results}

Pulmonary melanoma stimulates PD-L1. Studies were undertaken to determine whether the expression of ICP molecules was altered by the spread of melanoma to the lung. In these experiments, 8-week-old C57BL/6 mice were challenged with freshly prepared B16-F10 (B16) melanoma cells or vehicle control, and the pulmonary expression of ICPs was evaluated 2 weeks later. These studies demonstrated that melanoma progression to the lung was associated with significantly increased expression of multiple ICP moieties, including PD-1, PD-L1, and PD-L2 (Figure $1 \mathrm{~A}$ and Supplemental Figure 1; supplemental material available online with this article; https://doi.org/10.1172/JCI137750DS1). This induction was not specific for the PD-1/PD-L1/PD-L2 axis, since LAG3 and TIM3 were similarly induced (Supplemental Figure 1). Among these events, the induction of PD-L1 mRNA and protein was particularly prominent in comparisons of lungs from B16- and vehicle-challenged mice (Figure 1, A and B). FACS analysis demonstrated that this enhanced expression of PD-L1 was seen in a variety of cells, including airway and alveolar epithelial cells $\left(\mathrm{CC}^{+} 0^{+}\right.$and $\mathrm{SP}-\mathrm{C}^{+}$cells, respectively), $\mathrm{CD}^{+} \mathrm{T}$ cells, and

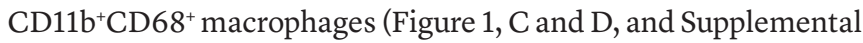
Figure 2 for gating strategy). PD-L1 expression in lung-resident $\mathrm{B}$ cells (CD19 ${ }^{+} \mathrm{B} 22 \mathrm{O}^{+}$cells) was not significantly altered by B16 tumor progression (Supplemental Figure 3). Double-label immunohistochemical staining using antibodies against cell-specific markers and PD-L1 further reinforced the enhanced expression of alveolar epithelial cell and macrophage PD-L1 (Figure 1E). These studies demonstrate that pulmonary melanoma metastasis is associated with significantly enhanced expression and accumulation of ICPs including PD-1/PD-L1 and PD-L2.

CHI3L1 plays a critical role in B16 melanoma stimulation of pulmonary PD-L1. Studies were next undertaken to define the potential role(s) of CHI3L1 in the induction of PD-L1 during the course of B16 tumor cell pulmonary progression. As noted above, the levels of mRNA encoding PD-L1 were significantly increased in lungs from melanoma-challenged mice compared with lungs from PBS controls (Figure 2A). This induction was significantly reduced in lungs from mice with null mutations of CHI3L1 (Figure 2A). Accordingly, PD-L1 protein accumulation was also increased in lungs from mice challenged with B16 cells compared with vehicle controls, and this induction was significantly decreased in lungs from CHI3L1null animals (Figure 2B). B16 cell stimulation of PD-L1 expression was also significantly diminished in lungs from mice treated with monoclonal anti-CHI3L1 antibody (called FRG antibody; Figure 2C). In accord with these findings, the ability of B16 cells to induce the accumulation of PD-L1 protein was also diminished by treatment with anti-CHI3L1 antibody (Figure 2D). These studies were reinforced by double-label immunohistochemical evaluations, which highlighted the impressive induction of PD-L1 in $\mathrm{CD}^{+}$ macrophages in lungs from B16-challenged mice and the decrease in PD-L1 accumulation in similarly challenged CHI3L1-null animals (Figure 2E). The importance of CHI3L1 in these inductive events was not unique to PD-L1, since null mutations of CHI3L1 and treatment with anti-CHI3L1 had similar effects on PD-1, LAG3, and TIM3 (Supplemental Figure 4). When viewed in combination, these studies demonstrated that CHI3L1 plays an essential role in melanoma stimulation of PD-L1 and other ICPs.

CHI3L1 plays an important role in local tumor progression and lymphatic spread. Although the B16-F10 tail vein injection model is commonly used in studies of tumor spread and metastasis, 


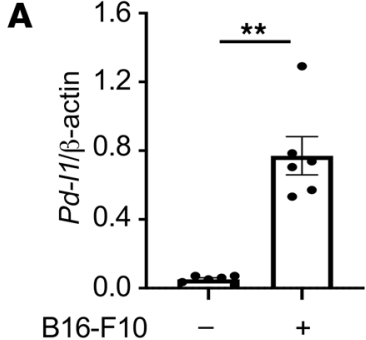

$\mathbf{B}$
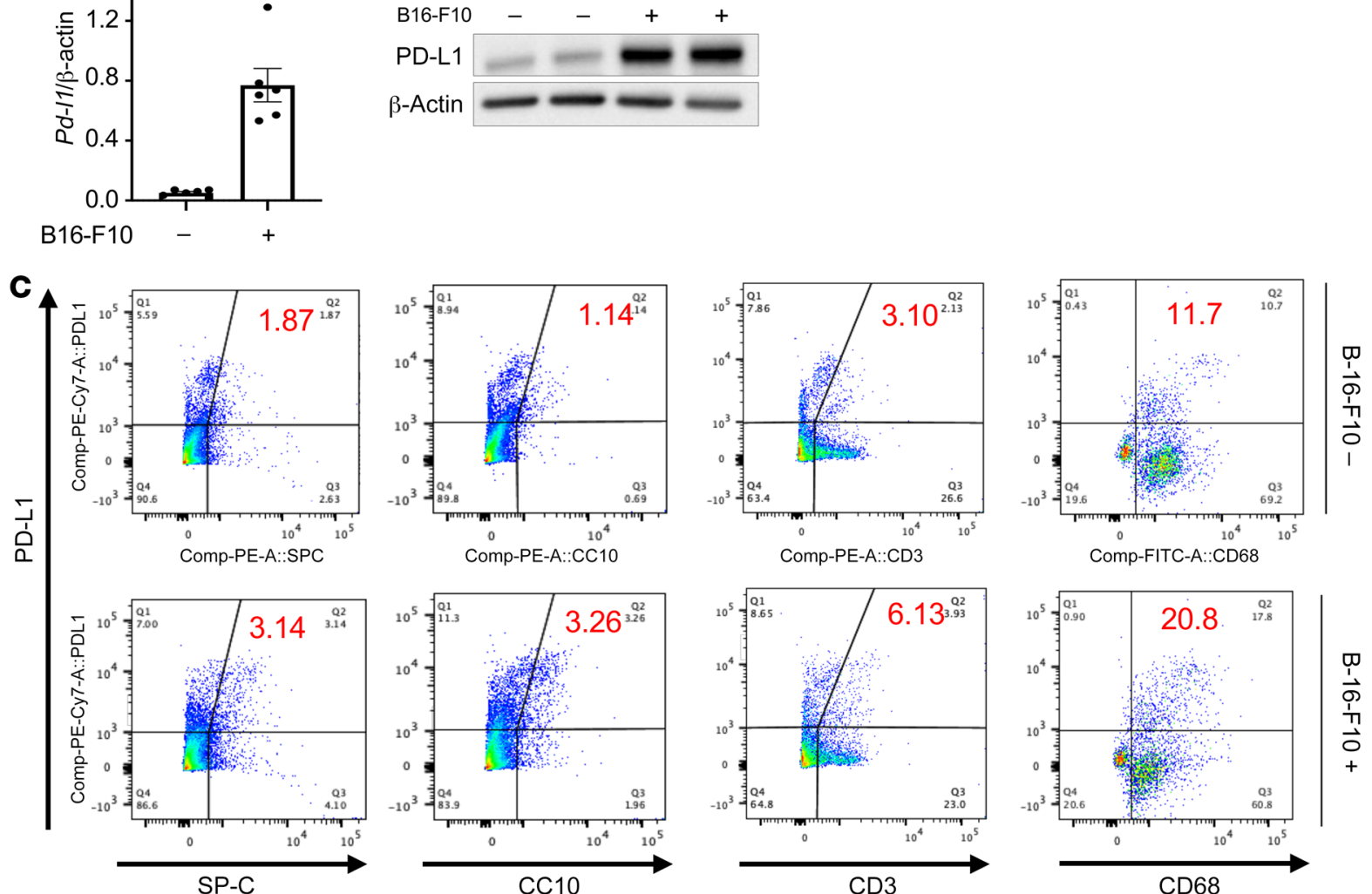

D
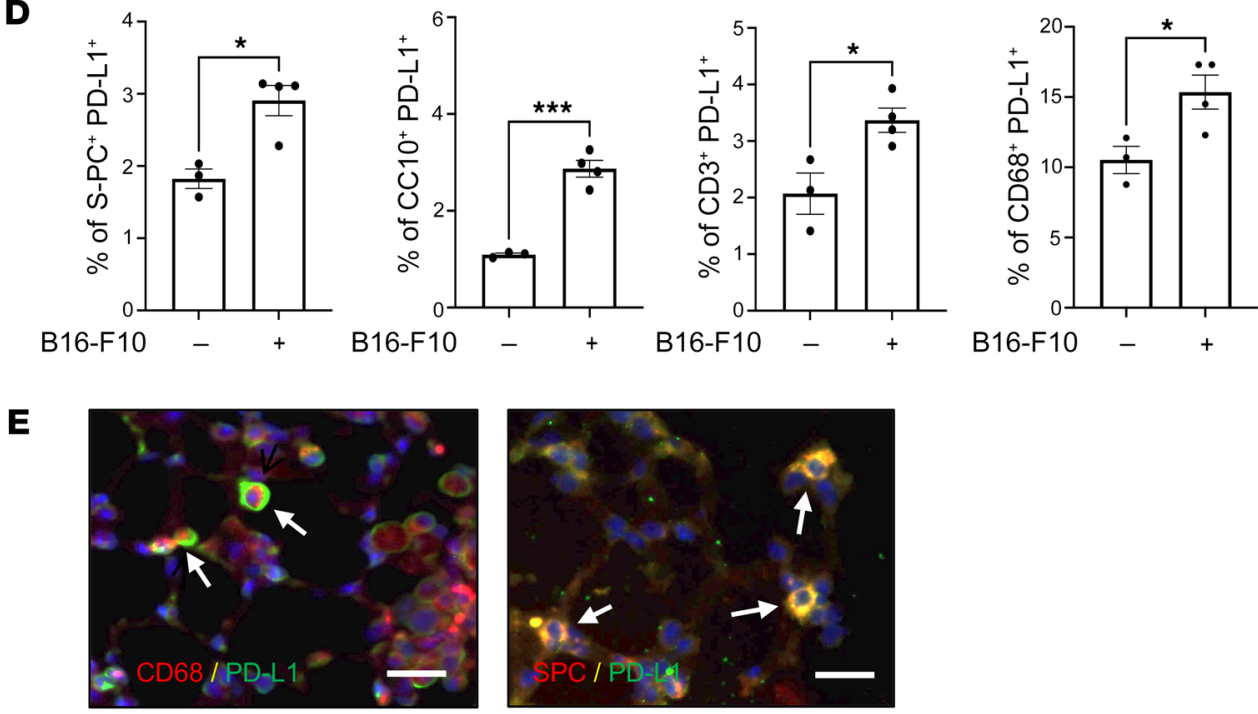

Figure 1. Pulmonary melanoma metastasis stimulates PD-L1. Eight-week-old WT mice were challenged with B16-F10 (B16) melanoma cells or control vehicle (PBS) via tail vein injection and evaluated 2 weeks later. (A) Real-time reverse transcriptase (RT) PCR was used to quantitate the levels of mRNA encoding PD-L1 in the lungs from mice treated i.v. with PBS (B16 -) or B16 cells (B16 +). Each dot represents an evaluation in an individual animal. (B) Western blot evaluations of PD-L1 accumulation in lungs from mice treated with PBS (B16 -) or B16 cells (B16 +). (C and D) FACS evaluations quantitating the accumulation of PD-L1 in cell populations from lungs of mice treated with B16 cells (B16-F10 +) or vehicle control (B16-F10 -). These evaluations used specific markers of airway epithelial cells (CC10), alveolar epithelial cells (surfactant apoprotein C [SP-C]), T cells (CD3), and macrophages (CD68). (E) Representative double-label fluorescent immunohistochemical evaluations in lungs from mice challenged with B16 melanoma cells using cell-specific markers (alveolar epithelial cells, SP-C; macrophages, CD68) (red) and anti-PD-L1 (green). The arrows highlight cells that stained with both antibodies. The values in $\mathbf{A}$ represent the mean \pm SEM of the noted evaluations represented by the individual dots. B-E are representative of a minimum of 2 similar evaluations. ${ }^{*} P<0.05$, ${ }^{*} P<0.01,{ }^{* * *} P<0.001$ (Student's $t$ test). Scale bars: $50 \mu \mathrm{m}$. 

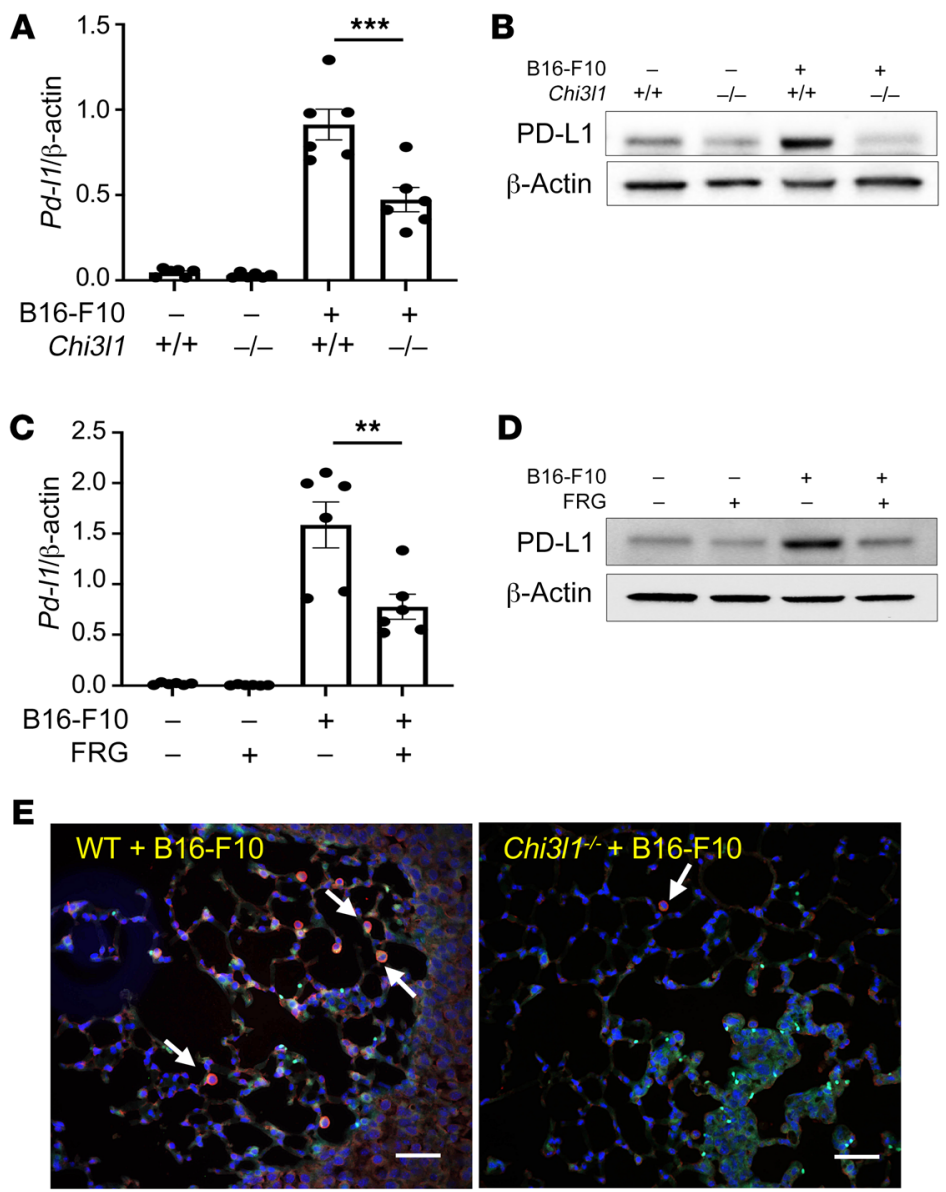

PD-L1/CD68/DAPI

others have proposed using more malignant melanocytes such as B16-BL6 cells in studies of tumor progression. In our studies with B16-BL6 cells, the cells were injected into a murine footpad $(2 \times$ $10^{4}$ cells per mouse), and the mice were randomized 2 weeks later to receive FRG or its antibody control $(200 \mu \mathrm{g} /$ mouse twice a week i.p.). Three weeks later, footpad tumor accumulation and popliteal lymph node size were assessed. As can be seen in Figure 3 , at the end of this 5-week experiment, FRG inhibited local tumor progression and the spread of tumor to popliteal lymph nodes. Thus, CHI3L1 plays an important role in local tumor progression and lymphatic spread.

Transgenic CHI3L1 stimulates PD-L1 in the normal lung. The studies noted above demonstrate that pulmonary melanoma metastasis stimulates PD-L1 and other ICPs and that CHI3L1 plays a critical role in these inductive events. However, because null mutations of CHI3L1 and treatment with anti-CHI3L1 decrease metastatic spread $(42,43)$, the studies do not determine whether the decreased induction of PD-L1 and other ICPs that is seen in CHI3L1-null mutant mice or mice treated with anti-CHI3L1 is due to the direct effects of CHI3L1 or the importance of CHI3L1 in melanoma spread. To address this issue, studies were undertaken to determine whether CHI3L1 stimulates pulmonary PD-L1 in the absence of B16 cell administration. In these experiments, we compared the expression and accumulation of PD-L1 in lungs from WT mice and transgenic mice in which CHI3L1 is overexpressed
Figure 2. CHI3L1 plays a critical role in B16 melanoma stimulation of pulmonary PD-L1. Eight-week-old WT $\left(\mathrm{Chi} / \mathrm{1}^{+/+}\right)$and CHI3L1-null $($ Chi3/1/-) $)$ mice were given B16 melanoma cells or vehicle control. They were also treated with an anti-CHI3L1 antibody (FRG) or isotype control antibodies, and PD-L1 expression was evaluated 2 weeks later. (A) RT-PCR was used to quantitate the levels of mRNA encoding PD-L1 in the lungs from mice treated i.v. with PBS vehicle (B16-F10 -) or B16 cells (B16-F10 +). WT and CHI3L1-null mice were used. Each dot represents an evaluation in an individual animal. (B) Western blot evaluations of PD-L1 accumulation in lungs from WT and CHI3L1-null mice treated with vehicle (B16-F10 -) or B16 cells (B16-F10 +). (C) RT-PCR was used to quantitate the levels of mRNA encoding PD-L1 in the lungs from mice treated i.v. with vehicle (B16-F10 -) or B16 cells (B16-F10 +). The mice were then treated with antibodies against CHI3L1 (FRG +) or isotype control antibodies (FRG -). Each dot represents an evaluation in an individual animal. (D) Western blot evaluations of PD-L1 accumulation in lungs from WT mice that were given control vehicle (B16-F10 -) or $\mathrm{B} 16$ cells $(\mathrm{B} 16-\mathrm{F} 10+)$ and treated with antibodies against anti-CHI3L1 (FRG +) or isotype control (FRG -) antibodies. (E) Double-label immunohistochemical comparison of lungs from WT and Chi3/1/- mice challenged with B16 melanoma cells using a macrophage-specific marker (CD68; green) and anti-PD-L1 antibodies (red). The plotted values in $\mathbf{A}$ and $\mathbf{C}$ represent the mean \pm SEM of the noted evaluations represented by the individual dots. B, D, and E are representative of a minimum of 2 similar evaluations. ${ }^{* *} P<$ $0.01,{ }^{* *} P<0.001$ (Student's $t$ test). Scale bars: $100 \mu \mathrm{m}$. in a lung-specific manner. These experiments demonstrated that CHI3L1 is a potent stimulator of PD-L1 mRNA and protein in lungs from transgenic mice compared with WT controls (Figure 4, A and B). FACS evaluations also demonstrated that transgenic CHI3L1 stimulated PD-L1 expression in macrophages (Figure 4, C and D). This effect was not PD-L1 specific, since transgenic CHI3L1 also stimulated PD-1, PD-L2, LAG3, and TIM3 (Supplemental Figure 5). These studies demonstrate that the stimulation of PD-L1 and other ICPs in pulmonary melanoma metastasis and spread is mediated, at least in part, by a tumor-independent effect of CHI3L1.

CHI3L1 stimulates pulmonary macrophage PD-L1. Because macrophages were the major cells expressing PD-L1 in response to transgenic CHI3L1, studies were undertaken to further understand the mechanism(s) of this inductive event. As can be seen in Figure $5 \mathrm{~A}$, the recovery of $\mathrm{CD} 45^{+} \mathrm{CD} 11 \mathrm{~b}^{+} \mathrm{CD} 68^{+} \mathrm{PD}-\mathrm{L} 1^{+}$cells was increased in lungs from CHI3L1-transgenic (YKL-40-transgenic) mice and decreased in lungs from CHI3L1-null animals compared with WT controls. In addition, the stimulatory effect of transgenic CHI3L1 (YKL-40) on $\mathrm{CD} 45^{+} \mathrm{CD} 11 \mathrm{~b}^{+} \mathrm{CD} 68^{+}$macrophages was significantly decreased in mice treated with anti-CHI3L1 antibody (FRG) compared with animals treated with an isotype antibody control (Figure 5B). We also compared the levels of mRNA encoding PD-L1 and PD-L1 protein in bone marrow-derived macrophages from WT mice treated with recombinant CHI3L1 or vehicle control. In these experiments recombinant CHI3L1 was a 


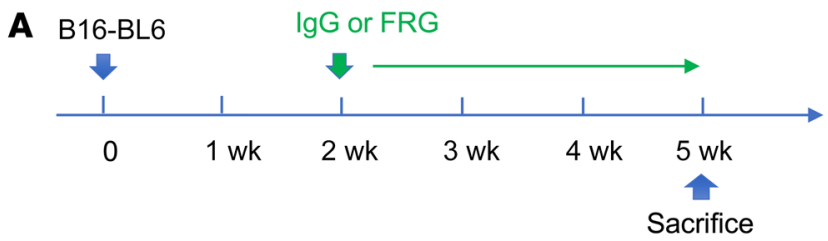

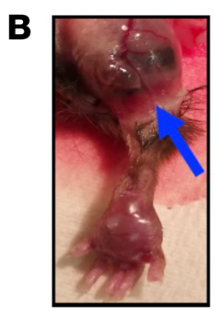

$\lg G$

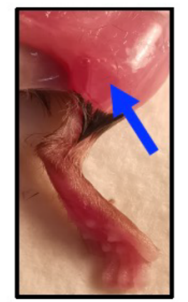

FRG

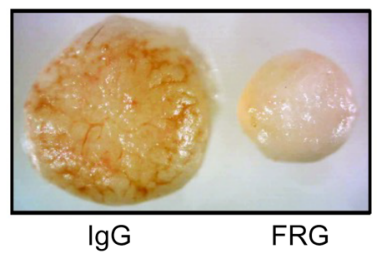

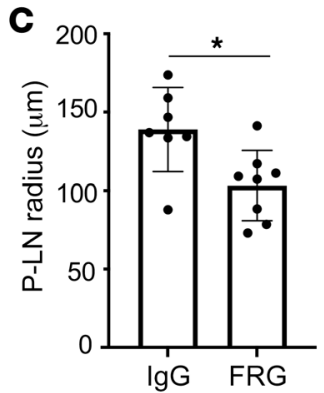

$\lg G \quad F R G$

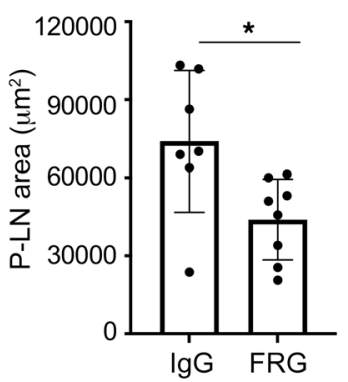

Figure 3. CHI3L1 regulates local footpad lymphatic spread of B16-BL6 cells. B16-BL6 cells $\left(2 \times 10^{4}\right)$ were injected into the right footpad. Two weeks later the mice were randomized to FRG or isotype control antibodies. After an additional 3 weeks the footpad lesions and popliteal lymph nodes were compared. (A) Treatment scheme used in these experiments. (B) Representative photographs of popliteal lymph nodes. The top panels compare the popliteal regions (indicated by arrows) of mice treated with control IgG versus FRG. The bottom panel compares popliteal lymph nodes from the right foot of mice treated with control IgG versus FRG. (C) Size (diameter and surface area) quantification of popliteal lymph nodes (P-LN). The values in $\mathbf{C}$ represent the mean \pm SEM of the noted evaluations represented by the individual dots. ${ }^{*} P<0.05$ (Student's $t$ test). potent, dose-dependent stimulator of PD-L1 mRNA and protein (Figure 5, C and D). PD-L2 was similarly stimulated (Figure 5E). These studies demonstrate that CHI3L1 stimulates the expression and accumulation of PD-L1 mRNA and protein in macrophages in vivo and in vitro.

CHI3L1 plays a critical role in IFN- $\gamma$ stimulation of macrophage $P D-L 1$. Previous studies reported that IFN- $\gamma$ is a potent stimulator of PD-L1 in macrophages and other immune cells $(45,46)$. Thus, studies were undertaken to define the role(s) of CHI3L1 in IFN- $\gamma$ stimulation of PD-L1. In these experiments, bone marrow-derived macrophages were obtained from WT and CHI3L1null mutant mice and were incubated with recombinant IFN- $\gamma$ (rIFN- $\gamma)$ or vehicle control for up to 72 hours. As shown in Figure $6 \mathrm{~A}, \mathrm{rIFN}-\gamma$ increased the levels of mRNA encoding PD-L1 in a time- and dose-dependent manner in WT cells (Figure 6, A and B). rIFN- $\gamma$ also stimulated macrophage CHI3L1 mRNA and protein expression in a dose- and time-dependent manner (Figure $6, \mathrm{C}$ and D). Interestingly, the levels of rIFN- $\gamma$-stimulated PD-L1 protein were significantly decreased in CHI3L1-null macrophages compared with macrophages from WT animals (Figure $6 \mathrm{E}$ ). A similar decrease in the number of macrophages expressing PD-L1 was seen in FACS evaluations of IFN- $\gamma$-stimulated cells from CHI3L1-null mice versus WT controls (Figure 6F). Similar CHI3L1 regulation of PD-L1 was noted in the lung-resident macrophages (Supplemental Figure 6). IFN- $\gamma$ also stimulated macrophage PD-L2 in a CHI3L1-dependent manner (Supplemental Figure 7). Null mutations of IL-13R $\alpha 2$ also significantly reduced IFN- $\gamma$ stimulation of macrophage PD-L1 (Figure 6G). In contrast, significant changes were not noted in the cells with null mutations of, or cells treated with inhibitors of, other putative CHI3L1 receptors or interacting partners such as TMEM219, galectin-3, or CRTH2 (Figure 6G and refs. 21, 23, 47). In combination, these studies demonstrate that CHI3L1 and IL-13R $\alpha 2$ play critical roles in IFN- $\gamma$ stimulation of macrophage PD-L1.
RLH activation inhibits CHI3L1 and PD-L1. We previously demonstrated that poly(I:C), a strong activator of retinoic acidinducible gene I (RIG-I) and the RIG-like helicase (RLH) innate immune response, prominently inhibits CHI3L1 expression and melanoma lung metastasis (43). Recent studies also demonstrated that RIG-I activation and mitochondrial antiviral signaling molecule (MAVS) are essential for many antitumor responses induced by ICP inhibitor blockade (44). Thus, studies were undertaken to determine whether RLH activation with poly(I:C) altered the ability of CHI3L1 to stimulate PD-L1. As shown in Figure 7A, poly(I:C) ameliorated melanoma-stimulated PD-L1 mRNA expression. Poly(I:C) similarly inhibited B16 cell stimulation of CHI3L1 and PD-L1 protein accumulation (Figure 7B). FACS analysis also demonstrated that macrophage expression of PD-L1 was prominently reduced by poly(I:C) treatment (Figure 7C). The suppressive effect on PD-L1 induced by poly(I:C) was at least partially dependent on CHI3L1, since the transgenic overexpression of CHI3L1 using a promoter that is not regulated by the RLH pathway ameliorated poly(I:C)-induced inhibition of PD-L1 and enhanced B16-stimulated expression of PD-L1 (Figure 7D). These studies highlight the ability of RLH activation to inhibit CHI3L1 and, in turn, inhibit PD-L1.

Anti-CHI3L1 and anti-PD-1 interact to augment antitumor responses. Since CHI3L1 stimulates $\mathrm{PD}-\mathrm{L} 1$ and other inhibitory ICP molecules, studies were undertaken to determine whether antiCHI3L1 and anti-PD-1 interact in inducing antitumor responses. In these experiments, mice were treated with the antibodies individually and in combination. As can be seen in Figure 8, FRG and anti-PD-1 individually inhibited melanoma progression in a dose-dependent manner when compared with isotype controls (Figure 8, A and B). Importantly, when mice were treated with $50 \mu \mathrm{g}$ doses of the 2 antibodies, the antitumor responses induced by the antibodies in combination exceeded the effects that were seen when the antibodies were used individually (Figure 8, A and 
A

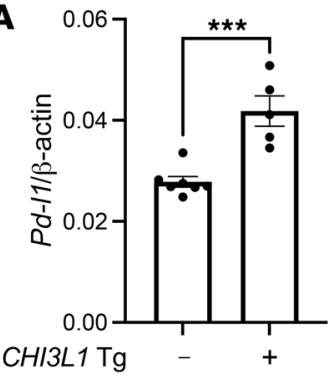

B

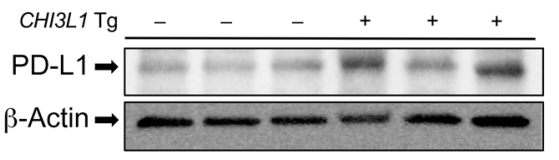

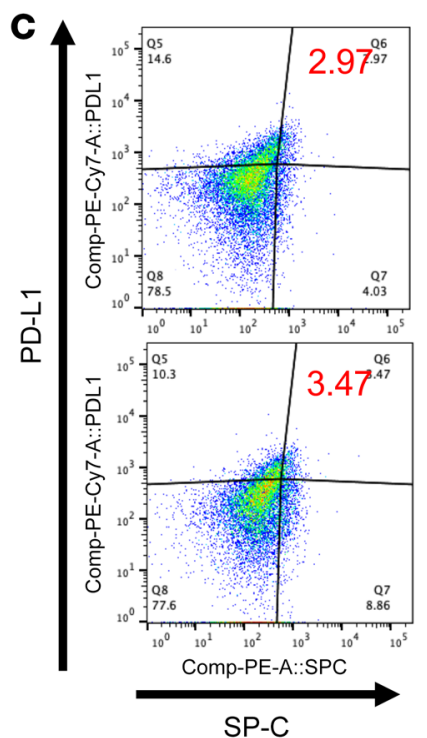
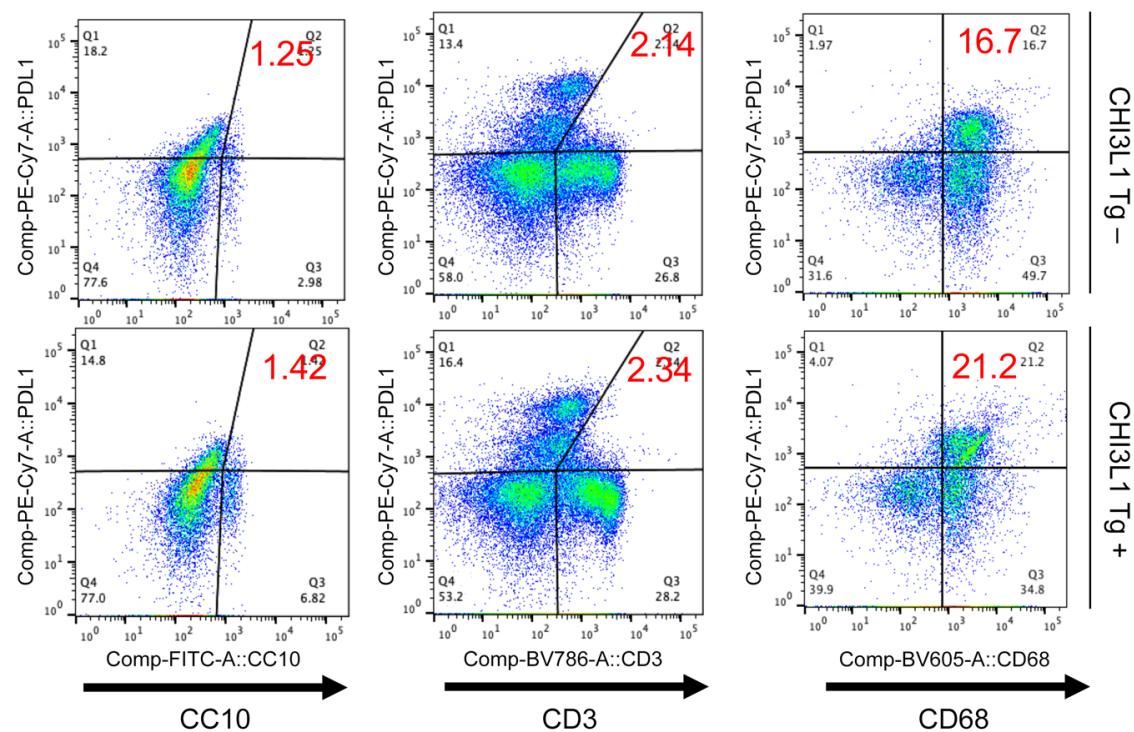

Comp-BV605-A::CD68

CD68
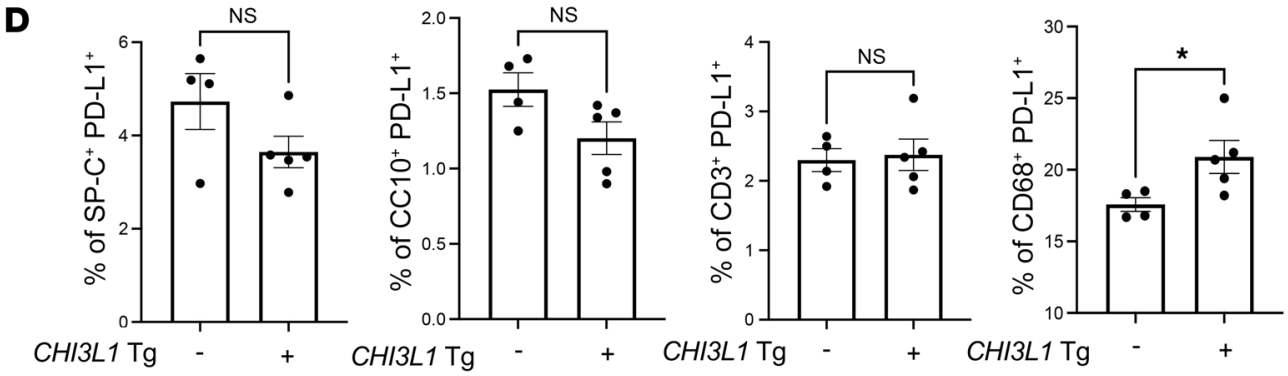

Figure 4. Transgenic CHI3L1 stimulates PD-L1 in the normal lung. Eight-week-old WT (-) and CHI3L1-transgenic (+) mice were used to evaluate the expression and accumulation of PD-L1 in the lung. (A) RT-PCR was used to quantitate the levels of mRNA encoding PD-L1 in the lungs from WT mice (CHI3L1 $\mathrm{Tg}-$ ) and mice in which CHI3L1 was overexpressed in the lung in a transgenic manner (CHI3L1 Tg +). Each dot represents the evaluation in an individual animal. (B) Western blot evaluations of PD-L1 accumulation in lungs from WT (CHI3L1 Tg -) and CHI3L1-transgenic (CHI3L1 Tg +) mice. (C and D) FACS evaluations quantitating the accumulation of PD-L1 in cell populations from lungs from WT and CHI3L1 Tg + mice. These evaluations used cell-specific markers of airway epithelial cells (CC10), alveolar epithelial cells (surfactant apoprotein C [SP-C]), dendritic cells (CD11c), and macrophages (F4/80). The values in A and $\mathbf{D}$ represent the mean \pm SEM of the noted evaluations represented by the individual dots. $\mathbf{B}$ is representative of a minimum of 2 similar evaluations. ${ }^{*} P<0.05,{ }^{* *} P<0.001$ (Student's $t$ test).

B). These effects appeared to be at least additive in nature. They demonstrate that anti-CHI3L1 and anti-PD-1 interact to augment antitumor responses in lung melanoma metastasis.

Bispecific antibodies that simultaneously target CHI3L1 and $P D-1$ synergistically induce CTL differentiation, PTEN expression, and tumor cell death. In recent years it has become clear that combination therapy with ICP blockers can induce particularly potent responses in a variety of tumors, including lung cancers (48-50). Recent studies have also demonstrated that bispecific antibodies can have powerful and/or unique biologic effects compared with their individual component antibodies, alone and in combination (51). The studies noted above suggest that antibodies against CHI3L1 and PD-1 interact to enhance antitumor responses in melanoma metastasis. Thus, studies were undertaken to determine whether bispecific antibodies that simultaneously target CHI3L1 and PD-1 elicit even more impressive antitumor responses. To address this question, we made bispecific antibodies in which anti-PD-1 was linked to the IgG1 Fc portion of FRG via its light chain (Supplemental Figure 8A). This antibody, termed FRGxPD-1, manifested high affinities to both CHI3L1 and PD-1 $\left(K_{D}=1.1 \times 10^{-9}\right)$ 
A

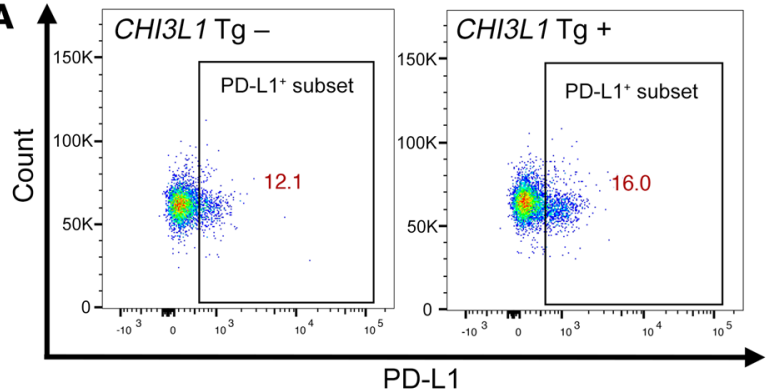

B

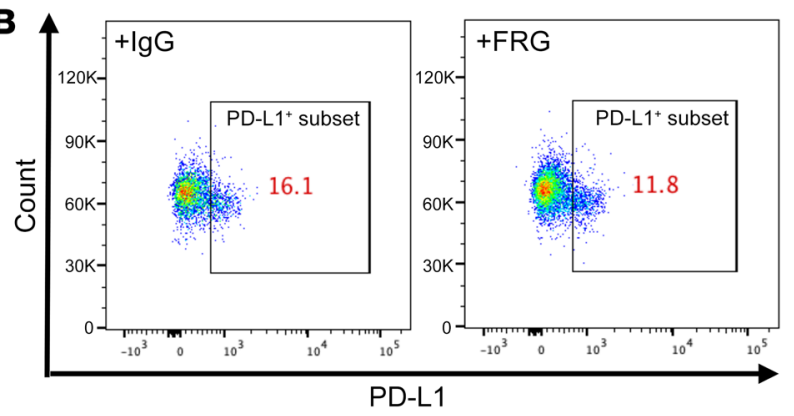

C

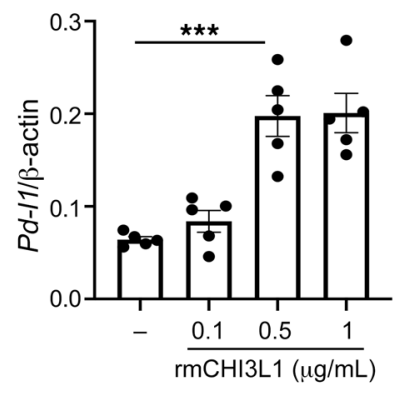

D

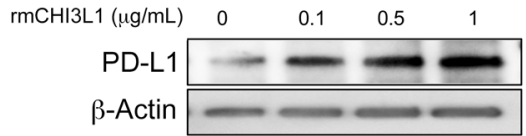

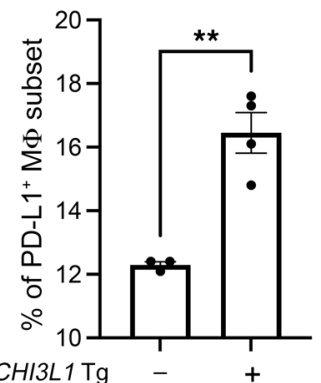

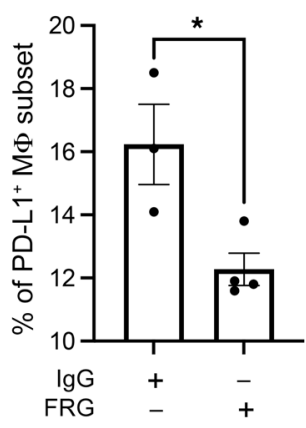

$\beta$-Actin $=\cdots$
Figure 5. CHI3L1 stimulates pulmonary macrophage PD-L1. Eight-week-old WT (-) and CHI3L1-transgenic (+) mice were used to evaluate macrophage-lineage cell PD-L1 in the lung. (A) FACS evaluations comparing PD-L1 on CD11 b+ cells isolated from lungs from WT versus CHI3L1-transgenic mice. (B) FACS evaluations comparing PD-L1 on $\mathrm{CD}_{68}{ }^{+}$cells isolated from lungs from WT mice treated with anti-CHI3L1 antibody (FRG) or its isotype control. (C) RT-PCR was used to quantitate the levels of mRNA encoding PD-L1 in bone marrow-derived macrophages from WT mice that were treated in vitro with recombinant murine (rm) CHI3L1 or vehicle control. Each dot represents an evaluation performed using cells from an individual animal. (D) Western blot evaluations of PD-L1 accumulation in bone marrow-derived macrophages from WT mice treated with the noted concentrations of rmCHI3L1 or vehicle control in vitro. (E) RT-PCR was used to quantitate the levels of mRNA encoding PD-L2 in bone marrowderived macrophages from WT mice after stimulation with the noted concentrations of rmCHI3L1 $(\mu \mathrm{g} / \mathrm{mL})$ or vehicle control in vitro. Each dot represents an evaluation performed using cells from an individual animal. $\boldsymbol{D}$ is representative of a minimum of 2 similar evaluations. The values in $\mathbf{C}$ and $\mathbf{E}$ represent the mean \pm SEM of the noted evaluations represented by the individual dots. ${ }^{*} P<0.05,{ }^{*} P<0.01,{ }^{* * *} P<0.001$ (A and $\mathbf{B}$, Student's $t$ test; $\mathbf{C}$ and $\mathbf{E}$, 1-way ANOVA with Tukey's post hoc test).

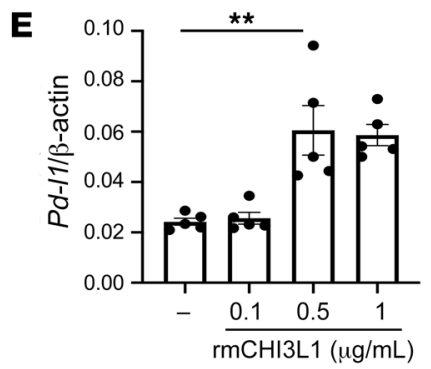

that were comparable to the affinities of the individual antibody moieties (Supplemental Figure 8B). We then compared the effects of FRGxPD-1 with those of FRG and anti-PD-1, alone and in combination, in a coculture system containing activated TALL-104 T cells and A357 human melanoma cells. In these coculture experiments, FRG and anti-PD-1 individually caused a significant increase in tumor cell apoptosis (Figure 9A). When FRG and anti-PD-1 were administered simultaneously, an additional increase in tumor cell death was seen. This effect appeared to be at least additive in nature (Figure 9A). Importantly, the highest levels of tumor cell apoptosis were seen when the bispecific antibody FRGxPD-1 was used (Figure 9A). This effect appeared to be synergistic in nature, since the levels of tumor cell death that were seen greatly exceeded the effects of FRG and anti-PD-1 when administered individually or in combination (Figure 9A). In all cases, the tumor cell death that was seen appeared to be mediated by $\mathrm{CD}^{+}$cytotoxic T cells, since FRG and anti-PD-1, alone and in combination, heightened $\mathrm{T}$ cell expression of $\mathrm{CD} 8$, perforin, and granzyme, and these effects were synergistically enhanced in cocultures treated with FRGxPD-1 (Figure 9, B-D). Surprisingly, FRG and anti-PD-1, alone and in combination, also heightened the expression of the tumor suppressor PTEN, and these effects were synergistically enhanced in cocultures treated with FRGxPD-1 (Figure 9E). Quantification of each evaluation is represented in Figure 9F. Identical results were seen in experiments that used these antibodies and the murine B16-F10 melanoma cell line (Supplemental Figure 8, C-G). When viewed in combination, these studies demonstrate that bispecific antibodies that simultaneously target CHI3L1 and PD-1 have impressive, synergistic antitumor effects that are mediated by their ability to induce CD8 ${ }^{+}$ cytotoxic T cells and enhance tumor cell expression of PTEN. 


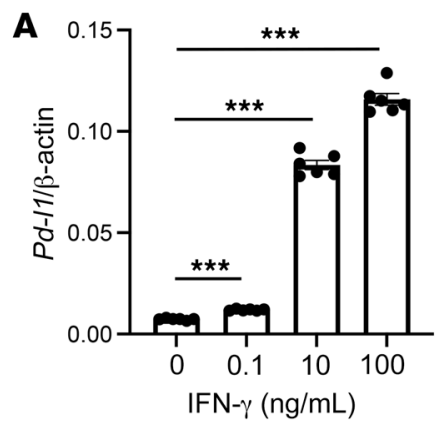

D
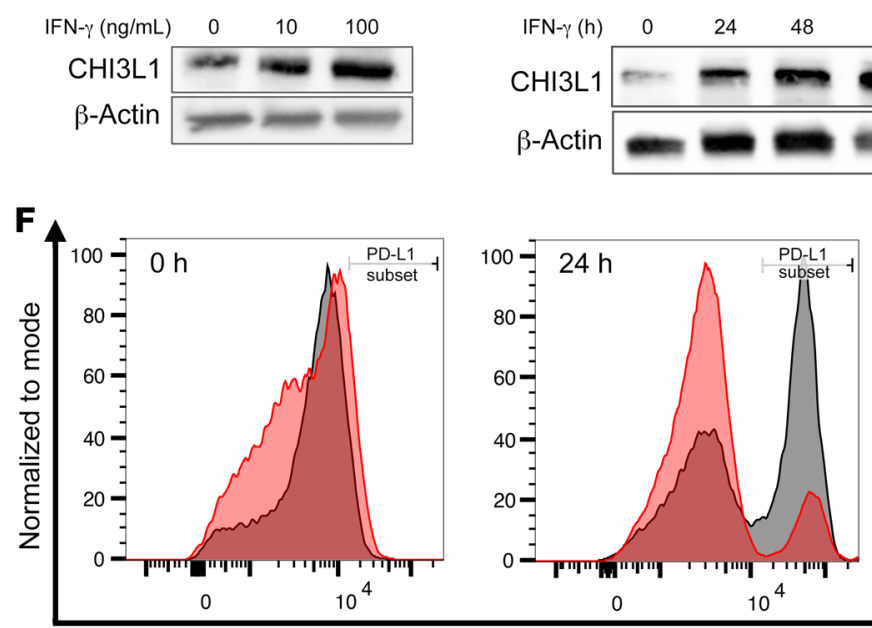

PD-L1 (IFN- $\gamma 100 \mathrm{ng} / \mathrm{mL}$ )
C
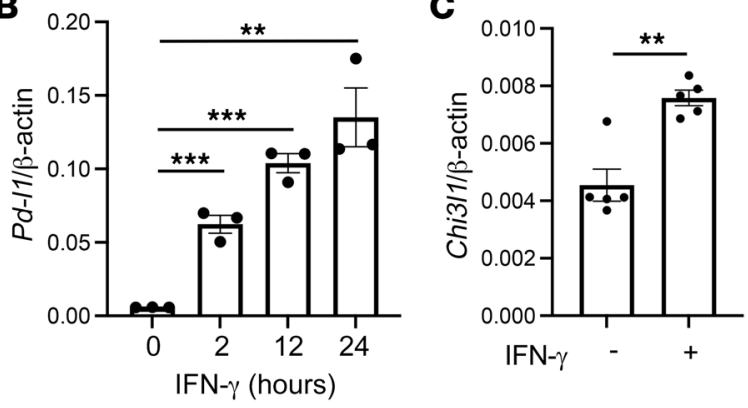

E

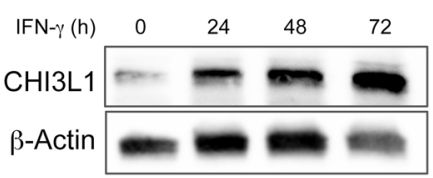

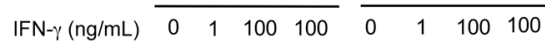

PD-L1

$\beta$-Actin

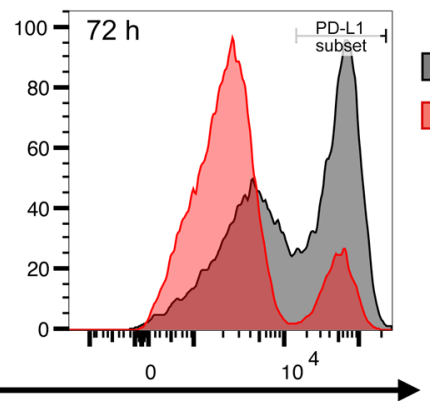

Chi3l1 $1^{+/ 4}$

Chi3/1-1-
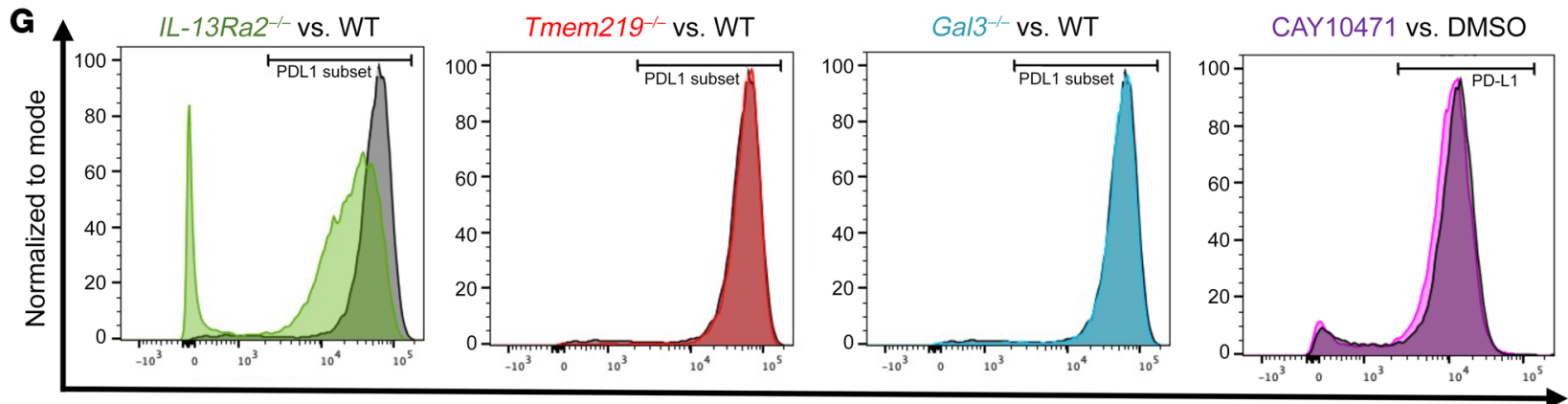

PD-L1 (IFN- $\gamma 100 \mathrm{ng} / \mathrm{mL})$

Figure 6. IFN- $\gamma$ stimulates macrophage PD-L1 via a CHI3L1-dependent mechanism. Bone marrow-derived macrophages (BMDMs) prepared from 6- to

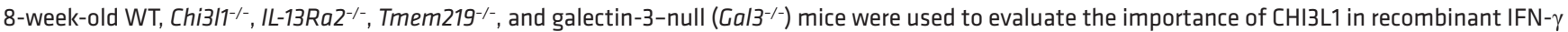
( $\mathrm{rIFN}-\gamma$ ) stimulation of PD-L1. (A and B) Dose and time dependency of IFN- $\gamma$ stimulation of PD-L1 mRNA in BMDMs. BMDMs from WT mice were incubated with the noted concentrations of rIFN- $\gamma$ for the noted periods of time. (C) RT-PCR evaluation of the expression of CHI3L1 in BMDMs after stimulation with rIFN- $\gamma$ for 24 hours. (D) Western blot evaluations of the dose and time dependency of IFN- $\gamma$ stimulation of macrophage CHI3L1 accumulation. (E) Western blot evaluations of rIFN- $\gamma$-stimulated PD-L1 accumulation in BMDMs prepared from WT and Chi3/1//- mice. (F) FACS evaluations of the ability of IFN- $\gamma$ to stimulate PD-L1 in BMDMs prepared from WT and Chi3/1/- mice. (C) FACS evaluations of the ability of IFN- $\gamma$ to stimulate PD-L1 accumulation in BMDMs

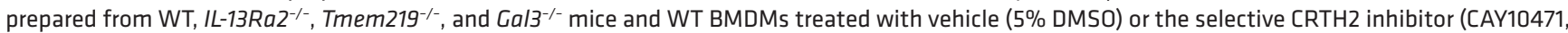
$20 \mu \mathrm{g} / \mathrm{mL}$ in $5 \% \mathrm{DMSO})$. The values in A-C represent the mean \pm SEM of the evaluations represented by the individual dots. D-C are representative of a minimum of 2 similar evaluations. ${ }^{* *} P<0.01,{ }^{* *} P<0.001$ (1-way ANOVA with Tukey's post hoc test).

Bispecific antibodies that simultaneously target CHI3L1 and PD-1 synergistically induce antitumor responses in vivo. The studies cited above demonstrate that the bispecific antibody that simultaneously targets CHI3L1 and PD-1 (FRGxPD-1) has synergistic tumor cytotoxic effects in the T cell coculture system when compared with the individual antibodies, alone and in combination. To see whether similar synergy was seen with this bispecific antibody in vivo, we compared the effects of FRGXPD-1 with the effects of the individual antibodies, alone and in combination. As can be seen in Figure 10, FRG and anti-PD-1 individually had discrete antitumor responses and interacted in an additive manner when combined in these assays. Importantly, even more impressive antitumor 
A

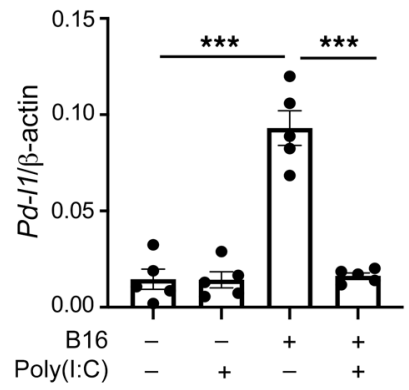

B

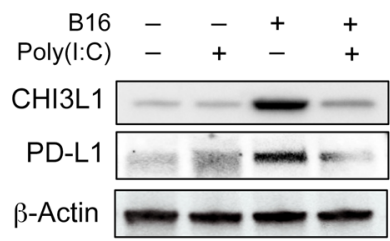

C

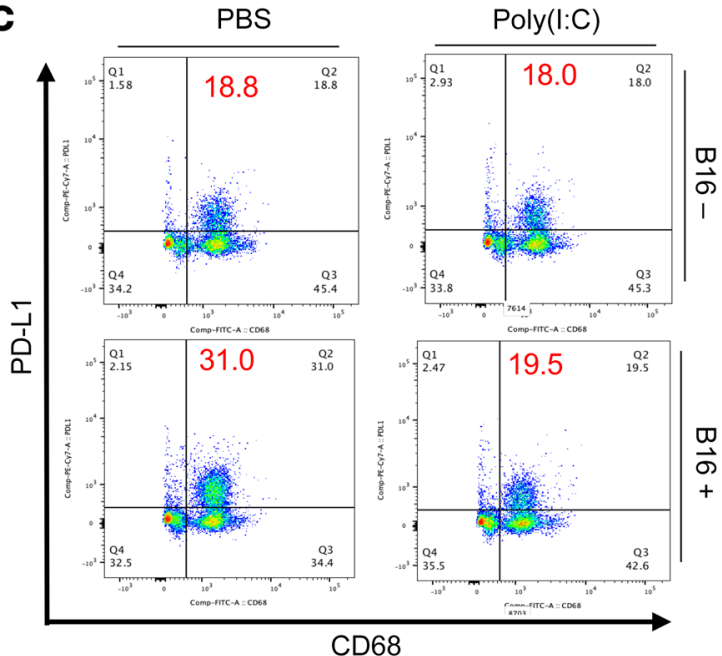

D

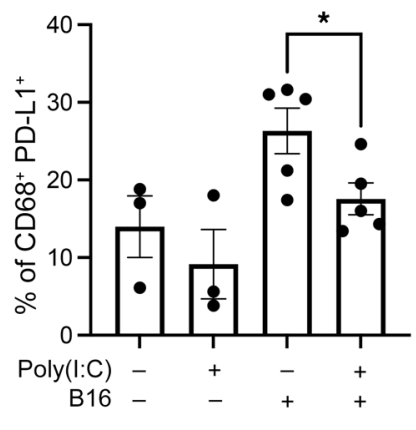

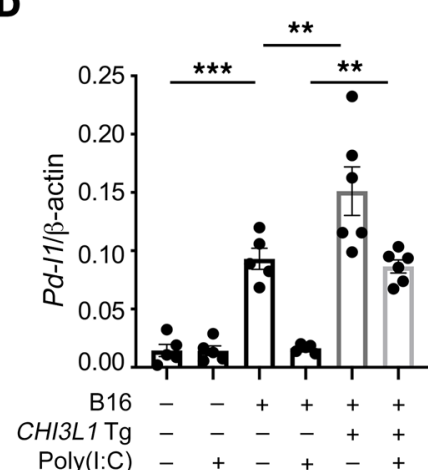

Figure 7. RIG-like helicase (RLH) activation inhibits the induction of CHI3L1 and PD-L1. WT mice were given B16-F10 (B16) melanoma cells or PBS control and treated with poly $(\mathrm{I}: \mathrm{C})$ or its vehicle control and evaluated 2 weeks later. (A and B) RT-PCR and Western evaluations were used to quantitate the levels of mRNA encoding PD-L1 and CHI3L1 and PD-L1 proteins in lungs from WT mice challenged with B16 cells (B16 +) or their PBS vehicle control (B16 -) that were treated with poly $(\mathrm{I}: \mathrm{C})$ or its vehicle control. (C) FACS evaluations of PD-L1 on CD68 ${ }^{+}$macrophages from lungs from WT mice that received B16 cells or their PBS controls and were treated with poly(I:C) or its vehicle control. (D) RT-PCR was used to quantitate the levels of mRNA encoding PD-L1 in the lungs from WT mice and CHI3L1 Tg + mice that were treated i.v. with vehicle (B16 -) or B16 cells (B16 +) and randomized to receive poly(I:C) or vehicle control. Each dot represents an evaluation in an individual animal. The plotted values in $\mathbf{A}$ and $\mathbf{D}$ represent the mean \pm SEM of the evaluations represented by the individual dots. $\mathbf{B}$ and $\mathbf{C}$ are representative of at least 3 similar evaluations. ${ }^{*} P<0.05,{ }^{* *} P<0.01,{ }^{* *} P<0.001$ (1-way ANOVA with Tukey's post hoc test).

responses were seen with FRGXPD-1, since it manifested antitumor responses that were significantly greater than what was seen with the individual antibodies alone or in combination.

\section{Discussion}

The immune surveillance hypothesis proposed in the 1950s that the immune system could recognize and reject cancer cells as foreign, in the same way that it reacts to microbes and transplanted organs (52). It has become clear over the last two decades that there is validity to this hypothesis and that tumors become shielded from immune elimination by aberrantly expressing ligands that normally interact with inhibitory immune receptors that protect "self" (52). To further understand the mechanisms that mediate these immunosuppressive responses, we tested the hypothesis that CHI3L1 plays a critical role in these tumor-inhibitory events. These studies highlighted the ability of pulmonary metastasis to stimulate components of the PD-1/PD-L1 axis and other ICP molecules. Importantly, they also highlighted a relationship between CHI3L1 and ICPs by demonstrating that CHI3L1 stimulates PD-L1, PD-1, PD-L2, and other ICPs in vivo and in vitro. In vivo, null mutations of or treatment with antibodies that target CHI3L1 abrogated the induction of PD-L1 and other ICPs by progressing tumors. Interestingly, transgenic CHI3L1, in the absence of tumor, reproduced these events, demonstrating that the effects of CHI3L1 are, at least partially, tumor independent. In vitro, CHI3L1 was shown to stimulate macrophage PD-L1, and IFN- $\gamma$ stimulation of PD-L1 was shown to be mediated, in part, by its ability to stimulate CHI3L1. These findings provide a potential mechanism(s) for the immunosuppressive effects of CHI3L1 that have been described in the context of breast cancer (53). They also describe a potentially important mechanism by which type I immune responses can be co-opted to shut down antitumor immune responses $(4,6)$. These studies also have important therapeutic implications because they demonstrate that the simultaneous administration of anti-CHI3L1 and anti-PD-1 antibodies generate at least additive antitumor responses in vivo. Importantly, they also demonstrate that bispecific antibodies that simultaneously target CHI3L1 and PD-1 synergistically induce $\mathrm{CD} 8{ }^{+} \mathrm{CTL}$ differentiation and tumor cell death in vitro and in vivo.

Early concepts of tumor immunity highlighted the importance of tumor cell expression of ligands like PD-L1 that bind to T cell PD-1 and suppress T cell activation (54). However, it is now clear 


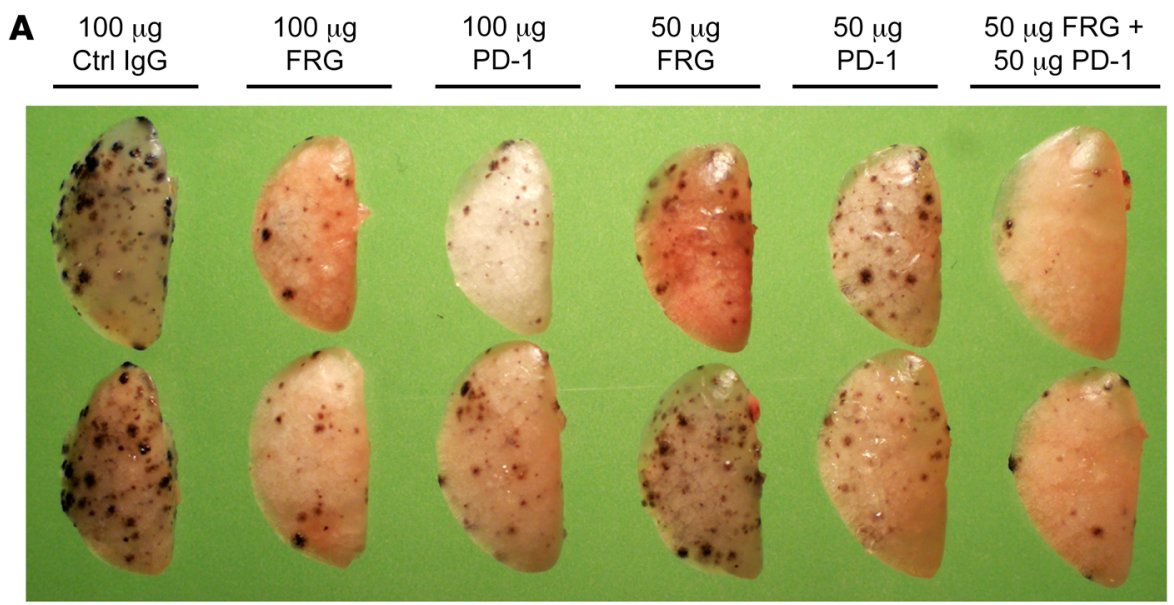

B

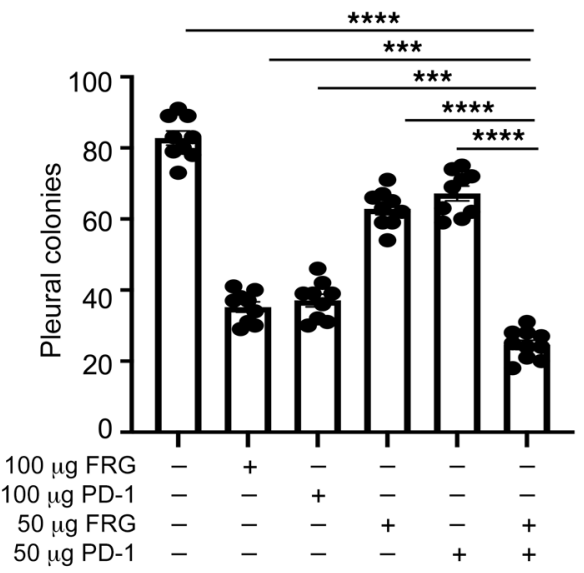

Figure 8. Anti-CHI3L1 and anti-PD-1 interact to augment antitumor responses in melanoma lung metastasis. WT mice were given B16-F10 melanoma cells or control vehicle and treated with control IgG, FRG, and/or anti-PD-1 antibodies, alone or in combination. Melanoma tumor burden was evaluated 2 weeks later. (A) Representative lungs from mice treated with control IgG, FRG, and/or anti-PD-1 antibodies, alone or in combination. As noted, the antibodies were given at doses of $100 \mu$ g every other day by i.p. injection from 1 day after melanoma cell challenge. (B) The number of pleural melanoma colonies was quantitated in the lungs from the mice in $\mathbf{A}$. Each dot is representative of an individual animal. $\mathbf{A}$ is representative of at least $\mathbf{3}$ similar evaluations. The values in $\mathbf{B}$ represent the mean \pm SEM of the evaluations represented by the individual dots in the lungs from the experiment representatively illustrated in $\mathbf{A} .{ }^{* *} P<0.001,{ }^{* * *} P<0.0001$ (1-way ANOVA with Tukey's post hoc test). that tumor-infiltrating immune cells can be the most common cells expressing PD-L1 in tumor specimens (6) and that tumor cell expression of PD-L1 does not always predict patient responsiveness to PD-1/PD-L1-based therapeutic interventions (6). In fact, in some settings the levels of PD-L1 on immune cells are a better predictor of responsiveness to anti-PD-1 than the levels of PD-L1 on tumor cells (6). In accord with this complexity, our studies demonstrate that metastatic B16-F10 cells and transgenic CHI3L1 stimulate PD-L1 in a variety of nontumor pulmonary cells. Macrophages were particularly prominent in these responses, and CHI3L1 was a powerful stimulator of macrophage PD-L1 in the murine lung and cultured bone marrow-derived macrophages. Studies of tumorassociated macrophages have demonstrated that some have an M1 (classic activation) phenotype. However, the majority appear to have an M2 (alternatively activated) phenotype with M2 cells predominating when the tumor metastasizes $(55,56)$. Previous studies from our laboratory and others have demonstrated that CHI3L1 contributes to macrophage diversity via the induction of M2 differentiation $(15,16,53)$. PD-L1 is now known to also play a regulatory role in M1/M2 polarization with PD-L1 increasing M2 differentiation (55). When viewed in combination, these studies allow for the overall hypothesis that CHI3L1 stimulates macrophage PD-L1, which augments the accumulation of M2 macrophages and generates a microenvironment that fosters tumor growth and metastasis.

IFN- $\gamma$ is a multifunctional type I cytokine that is known to induce antitumor responses via a number of mechanisms, includ- ing the activation of macrophages and monocytes (46). In accord with this concept, the secretion of IFN- $\gamma$ by stimulated blood mononuclear cells from patients with advanced cancer has been reported to be significantly decreased in comparison with cells from healthy controls $(46,57)$. In contrast, in certain settings, IFN- $\gamma$ also acts to induce tumor progression (46). Although the mechanisms that underlie the tumor-permissive effects of IFN- $\gamma$ have not been fully defined, recent studies suggest that IFN- $\gamma$ does this, in part, by inducing immune escape via the induction of PD-L1 (46). Our studies support this concept by demonstrating that IFN- $\gamma$ stimulates PD-L1 in macrophages and other cells. They also demonstrate that IFN- $\gamma$ stimulation of CHI3L1 is a critical event in this PD-L1-inductive response. To enhance the antitumor effects of IFN- $\gamma$, it has been suggested that rIFN- $\gamma$ be administered to patients with low IFN- $\gamma$ activity and that anti-PD-1/PD-L1 be coadministered in these circumstances $(46,57)$. Our studies add to this concept by suggesting that patients who are being treated with IFN- $\gamma$ or who have high levels of IFN- $\gamma$ activity could be treated with CHI3L1 inhibitors or FRGxPD-1 to maximize the therapeutic efficacy of IFN- $\gamma$. Further experimentation will be required to address these possibilities.

Because CHI3L1 lacks enzymatic activity when compared with true chitinases, studies were undertaken to determine whether it mediated its functions via novel receptors. These studies demonstrated that CHI3L1 binds to, signals, and confers tissue responses via IL-13R $\alpha 2$ and CRTH2, a known prostaglandin $\mathrm{D}_{2}$ receptor 
$\lg G$

A

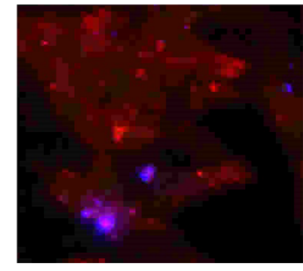

B

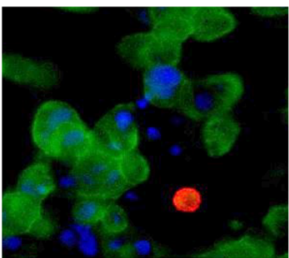

C

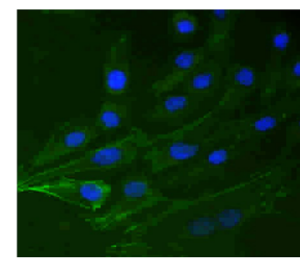

D

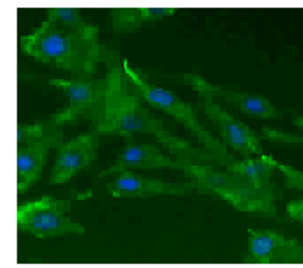

E

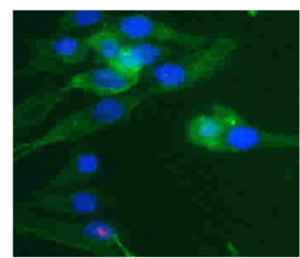

F

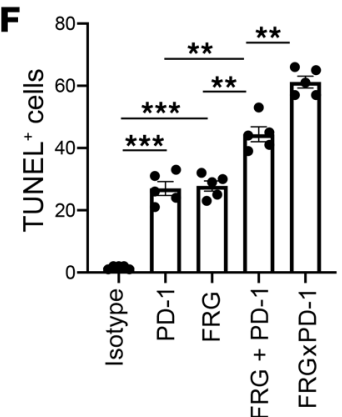

PD-1
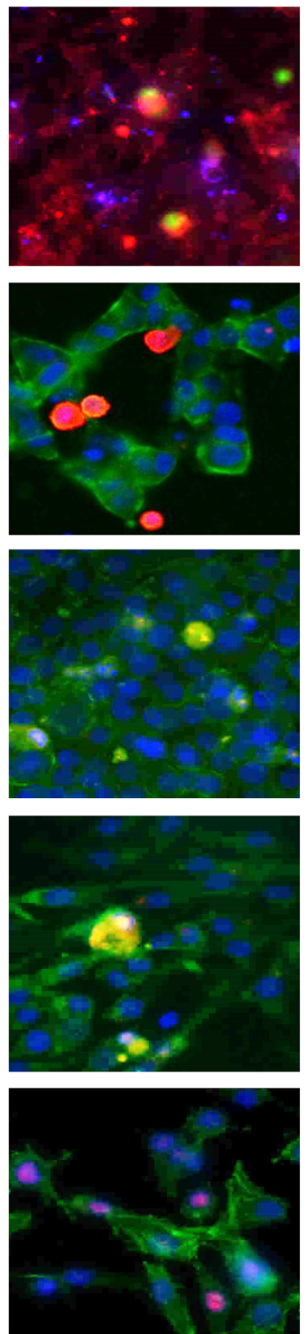

FRG
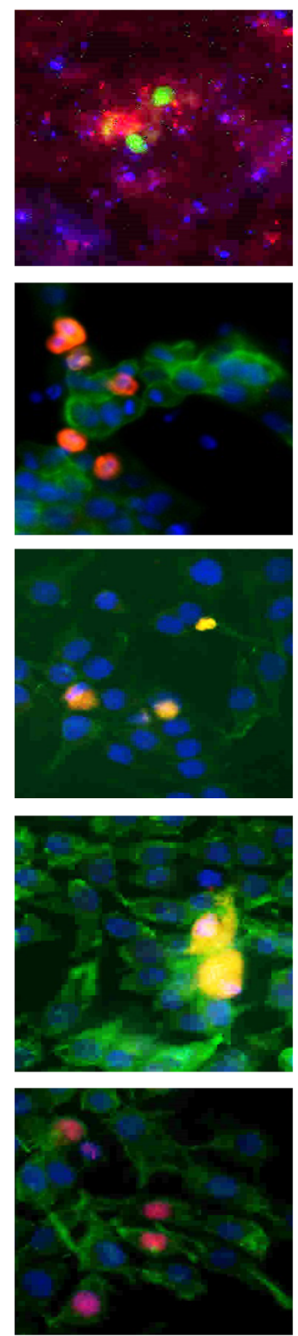

$F R G+P D-1$
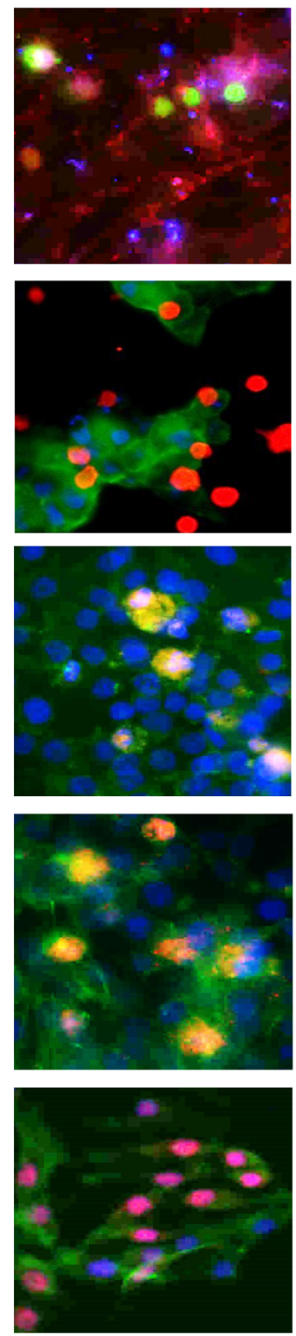

FRGXPD-1
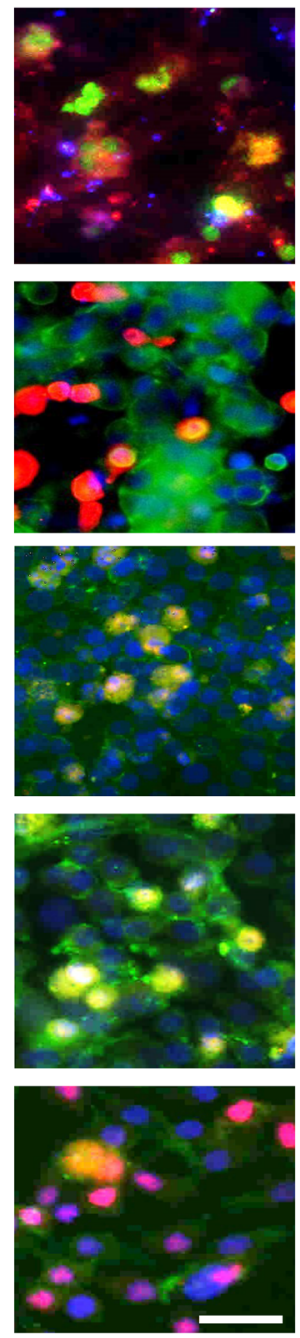
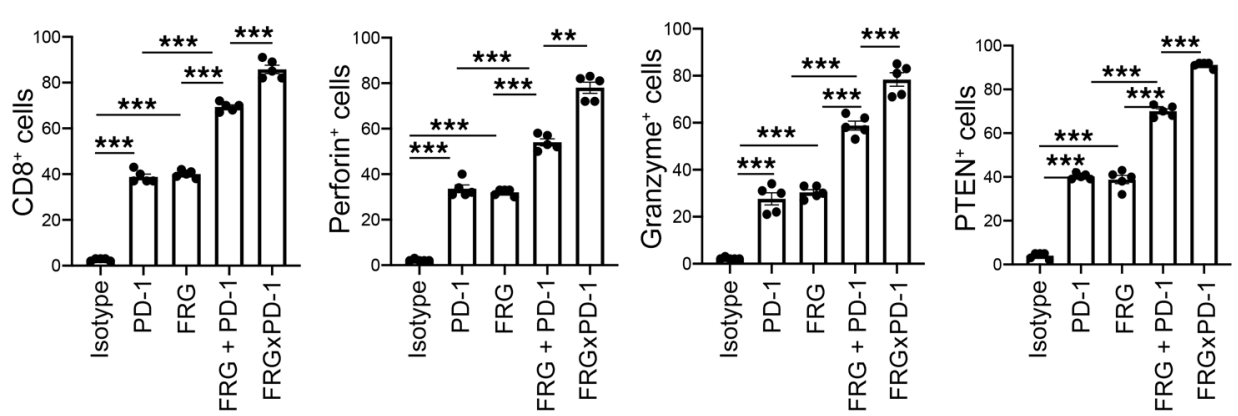

Figure 9. Bispecific antibodies that simultaneously target CHI3L1 and PD-1 induce synergistic CTL-mediated tumor cell death responses and tumor cell PTEN expression. The antitumor effects of the FRGXPD- 1 bispecific antibody were evaluated in a coculture system containing TALL-104 cells and A375 human melanoma cells. TALL-104 cells were activated by pretreatment with anti-CD3 and anti-CD28 $\left(1 \mu \mathrm{g} / \mathrm{mL}\right.$ each; incubation for 2 hours in $5 \%$ CO ${ }_{2}$ and air at $\left.37^{\circ} \mathrm{C}\right)$. The TALL-104 cells were then cocultured with A357 human melanoma cells for 24 hours. These cocultures were undertaken in the presence of the following antibodies: isotype control antibody $(5 \mu \mathrm{g} / \mathrm{mL})$, anti-PD-1 or anti-CHI3L1 (FRG) alone ( $5 \mu \mathrm{g} / \mathrm{mL}$ ) or in combination ( $2.5 \mu \mathrm{g} / \mathrm{mL}$ each), and the bispecific FRGXPD-1 antibody ( $5 \mu \mathrm{g} / \mathrm{mL}$ ). (Row A) Representative demonstration of apoptotic tumor cell death using the In Situ Cell Death Detection Kit with fluorescein-dUTP. TUNEL+ cells are stained green. (Rows B-D) Representative demonstration of TALL-104 T cell expression of CD8 (B), perforin (C), and granzyme (D). Tumor cells are green, and positive-staining TALL-104 cells are yellow-orange. (Row E) Representative demonstration of tumor cell PTEN. Tumor cells are green, and PTEN is yellow-orange. (F) Quantification of the evaluations in A-E. The percentage of TUNEL tumor cells (row A), percentage of TALL-104 cells expressing CD8 (row B), perforin (row C), and granzyme (row D) adherent to tumor cells, and percentage of tumor cells expressing PTEN (row E) are illustrated. These evaluations were done using fluorescent microscopy (original magnification, $\times 20$ ). In these quantifications, 5 randomly selected fields were evaluated. The values in $\mathbf{F}$ are the mean \pm SEM of the noted 5 evaluations. ${ }^{* *} P<0.01,{ }^{* * *} P<0.001$ (1-way ANOVA with Tukey's post hoc test). Scale bar: $10 \mu \mathrm{m}$ (applies to all subpanels of $\mathbf{A}-\mathbf{E})$. 
A $\lg G$

FRG

Anti-PD-1 (PD-1)

Anti-FRG + PD-1 (FRG+PD-1)

Anti-FRGxPD-1 (FRGXPD-1)

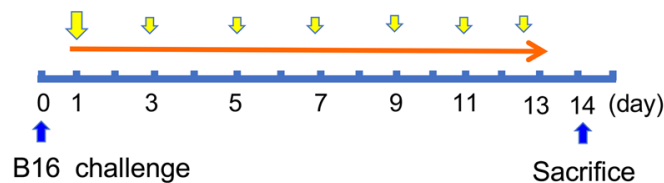

B

$\begin{array}{ccccc}\text { Ctrl lgG } & \text { FRG } & \text { PD-1 } & \text { FRG + } & \text { FRGx } \\ (100 \mu \mathrm{g}) & (100 \mu \mathrm{g}) & (100 \mu \mathrm{g}) & \mathrm{PD}-1 & \mathrm{PD}-1\end{array}$

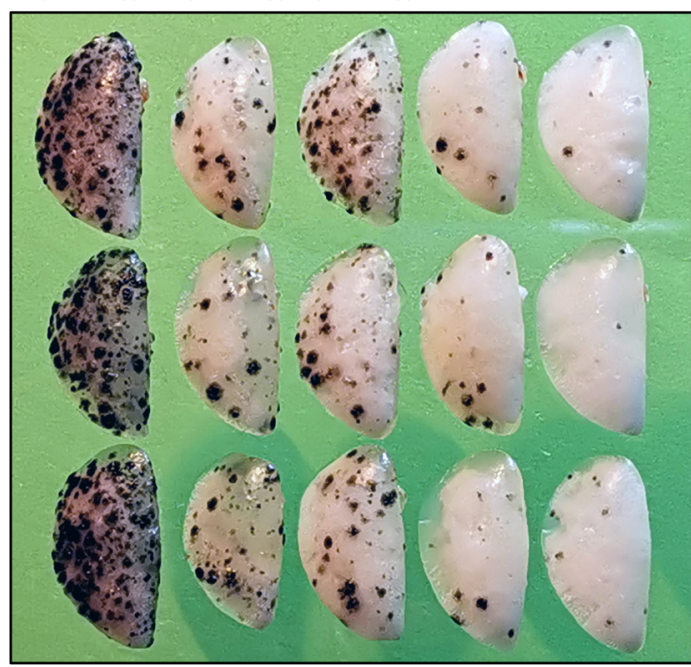

Figure 10. Bispecific antibodies that simultaneously target CHI3L1 and PD-1 induce synergistic antitumor responses. WT mice were given B16-F10 (B16) melanoma cells or control vehicle and treated with control IgG, FRG, and/or anti-PD-1 antibodies (alone or in combination) and bispecific FRGXPD-1 antibody. Melanoma lung metastasis was evaluated 2 weeks later. (A) Schematic illustration of antibody treatment protocol in the course of B16 melanoma cell challenge and mouse sacrifice. (B) Representative lungs from mice treated with control IgG, FRG, and/or anti-PD-1 antibodies (alone or in combination) and bispecific FRGXPD-1 antibody. The antibodies were given at the noted doses by i.p. injection. (C) The number of pleural melanoma colonies was quantitated in the lungs from the mice in $\mathbf{A}$. Each dot is representative of an individual animal. $\mathbf{B}$ is representative of at least 3 similar evaluations. The values in $\mathbf{C}$ represent the mean \pm SEM of the evaluations represented by the individual dots in the lungs from the experiment representatively illustrated in B. ${ }^{*} P<0.05$, ${ }^{* *} P<0.001$ (1-way ANOVA with Tukey's post hoc test).

$(18,23)$. They also demonstrated that IL-13R $\alpha 2$ plays a central role in a multimeric receptor complex called the chitosome that has an IL-13R $\alpha 2$ subunit and at least one $\beta$ subunit called TMEM219 (TMEM) $(18,21)$. The other $\beta$ subunit may be CD44 (variant 3), which is now known to bind IL-13R 22 (58). These studies also demonstrated that CHI3L1 activates the MAPK, Akt/protein kinase $\mathrm{B}$, and Wnt/ $\beta$-catenin signaling pathways with optimal MAPK and Akt activation requiring IL-13R $\alpha 2$ and TMEM and Wnt/ $\beta$-catenin signaling being mediated via a TMEM-independent mechanism $(18,21)$. To further understand the mechanisms by which CHI3L1 regulates $\mathrm{PD}-\mathrm{L} 1$, we compared the ability of $\mathrm{CHI}$ L1 to stimulate PD-L1 in macrophages from WT mice, mice with null mutations of IL-13R $\alpha 2$ or TMEM, and WT cells treated with a specific CRTH2 inhibitor. These studies demonstrated that IL-13R $\alpha 2$ plays a critical role in CHI3L1 stimulation of macrophage PD-L1. In contrast, CHI3L1 induction of PD-L1 was not altered by null mutations of TMEM or by treatment with the CRTH2 inhibitor. These obser- vations raise the interesting possibility that Wnt $/ \beta$-catenin pathway activation plays a major role in this CHI3L1 stimulatory event. We cannot, however, rule out contributions from MAPK or Akt signaling, because they have been implicated in the regulation of PD-L1 in other circumstances.

Activation of the RIG-I/RLH signaling pathway in tumors and the host microenvironment has recently been demonstrated to be a critical component of immune checkpoint blockade (ICB) induced by antibodies against CTLA4, alone and in combination with antibodies against PD-1 (44). When RIG-I/RLH innate immunity is appropriately induced, ICB triggers caspase-3mediated tumor cell death, cross-presentation of tumorassociated antigen by $\mathrm{CD} 103^{+}$ dendritic cells, and the accumulation of antigen-specific $\mathrm{CD}^{+}$ infiltrating $\mathrm{T}$ cells (44). It was speculated that nucleic acids leaking from disintegrating tumor cells during RIG-I-induced cell death are engulfed by host myeloid cells to induce RLH activation and type I IFN production (44). We previously demonstrated that RLH activation inhibits CHI3L1 production (43). The present studies demonstrate that RLH inhibition of CHI3L1, in turn, inhibits PD-L1 and other ICPs. They also demonstrate that at least additive antitumor effects are seen when mice with B16-F10 melanoma metastasis are treated simultaneously with anti-CHI3L1 and anti-PD-1 individually and that synergistic tumor cell death responses are seen in tumor-T cell cocultures treated with the bispecific antibody FRGxPD-1. These studies provide an additional mechanism that can explain how RIG-I/RLH activation fosters ICB antitumor responses. Specifically, they suggest that the antitumor effects of RIG-I/RLH activation are due to its ability to inhibit CHI3L1 and that CHI3L1 is a critical regulator of ICB. This raises the exciting possibility that interventions that inhibit or block CHI3L1 can be used to maximize the therapeutic efficacy of ICB.

LAG3 was cloned in 1990 and shown to have 20\% homology with CD4 and bind to and inhibit T cell activation via MHC class II. It is upregulated as a feedback mechanism on activated and exhausted T cells (4). Our studies define, for the first time to our knowledge, a relationship between LAG3 and CHI3L1 by demon- 
strating that CHI3L1 is a potent stimulator of LAG3. In so doing they demonstrate that the effects of CHI3L1 on ICPs are not specific for components of the PD-1/PD-L1 pathway. They also provide a potential explanation for the synergy between interventions that target CHI3L1 and PD-1, because LAG3 and PD-1 are known to interact synergistically to suppress $\mathrm{T}$ cell activation $(4,59)$.

Lung cancer is the leading cause of cancer-related mortality worldwide (60). In keeping with its importance, our knowledge of tumor immunology and ICP inhibitors has led to the development of a number of new therapeutics that have been assessed in metastatic and primary lung cancers (52). In contrast to the prior therapeutic dogma that focused initially on tumor resection followed by chemotherapy and/or radiation therapy, ICP inhibitors are now first- or second-line therapeutics (61). When these agents are used, remarkable responses are seen in some individuals. However, overall, only a minority of patients respond to these therapies, and the responses that are seen are often not durable (62). To address these issues, combination immunotherapy has been attempted. The simultaneous treatment with anti-PD-1 and anti-CTLA4 has been most commonly employed (63). This combination has engendered interesting effects in some malignancies. However, it has not proven successful in lung cancers. In addition, its use is limited by toxicities such as the pneumonitis that is caused by the exaggerated immune response that the antibody combination induces (63). In keeping with the concept that combination immunotherapy may be therapeutically useful, we evaluated the effects of anti-CHI3L1 (FRG) and anti-PD-1, alone and in combination. These studies demonstrate that the simultaneous treatment with antibodies that target CHI3L1 and PD-1 individually results in additive antitumor responses. Based on our finding that RLH activation inhibits CHI3L1, we can also envision similarly augmented anticancer response if RLH activators and anti-PD-1 are coadministered. Whether the safety profile of these immunotherapeutic combinations is better or worse than that of anti-PD-1 plus anti-CTLA4 will need to be determined.

Bispecific antibodies are a growing class of immunotherapies with the potential to further improve clinical efficacy and safety (51). In some cases, the targeted moieties redirect cytotoxic effectors. In others they simultaneously target immunomodulators and tumor antigens (51). Most recently, bispecific antibodies have been generated that simultaneously target more than one immunomodulatory pathway (reviewed in ref. 51). To address the possibility that bispecific antibodies that simultaneously target CHI3L1 and PD-1 might be particularly effective, bispecific antibodies were generated and their effects in vivo and in a T cell-melanoma cell coculture system were evaluated and compared with the effects of individual antibodies, alone and in combination. These studies demonstrated that FRGxPD-1 was a remarkably effective inducer of tumor cell death in vitro and in vivo. They also demonstrated that these antitumor responses were synergistic when compared with the effects of the individual antibodies. Lastly, they demonstrated that the tumor cell death responses were due to the ability of FRGxPD-1 to induce CD8 ${ }^{+}$, perforin ${ }^{+}$, and granzyme $^{+}$cytotoxic $\mathrm{T}$ cells. These findings agree with a fundamental principle of tumor immunology, that cancer cells can be eliminated by host cytotoxic T cells (64). The synergistic nature of the responses induced by FRGxPD-1 further supports our contention that multiple different immunoregulatory pathways are involved in this response. In addition to the ability of anti-PD-1 to block the interactions of PD-1 with PD-L1 and/or PD-L2, interventions that diminish the production and/or effector functions of CHI3L1 inhibit the ability of CHI3L1 to inhibit cell death (apoptosis and pyroptosis), and augment the accumulation of IFN- $\alpha / \beta$, chemerin and its receptor ChemR23, phosphorylated cofilin, and LimK2 (43). CHI3L1-based interventions also augment the expression of the tumor suppressor PTEN, and decrease M2 macrophage differentiation and augment type I immune responses (43). One can understand how the effects on PTEN contribute to tumor control because PTEN is a tumor suppressor whose functional loss leads to the activation of PI3K/Akt signaling, the inhibition of apoptosis, abnormal cell proliferation, angiogenesis, and melanoma development, invasion, and progression (65). When viewed in combination, these studies demonstrate that bispecific antibodies that simultaneously target CHI3L1 and PD-1 have impressive synergistic antitumor effects while simultaneously inducing CD8 ${ }^{+} \mathrm{CTL}$ differentiation and tumor cytotoxicity. When viewed in combination, these findings highlight the potentially exciting therapeutic effects of this antibody construct.

In conclusion, these studies demonstrate that CHI3L1 stimulates PD-L1 and other ICP moieties in vivo and in vitro. They also demonstrate that the simultaneous treatment of progressive pulmonary melanoma with individual antibodies against CHI3L1 and PD-1 generates an additive antitumor response and that bispecific antibodies that simultaneously target CHI3L1 and PD-1 synergistically induce antitumor responses in vivo and CD8 ${ }^{+}$CTL-mediated tumor cell death. These findings predict that interventions that simultaneously target CHI3L1 can augment the efficacy of ICP blockade in response to anti-PD-1 in lung cancer and potentially other malignancies. Additional investigations of the biology and therapeutic consequences of interactions between CHI3L1 and ICP inhibitors are warranted.

\section{Methods}

Genetically modified mice. Mice with null mutations of CHI3L1 (Chi3l1 $1^{-/}$) and TMEM219 (Tmem219/-) and transgenic mice in which human CHI3L1 was targeted to the lung with the CC10 promoter (CHI3L1-Tg) were generated and characterized by our laboratory as previously described $(16,21)$. Mice with null mutations of IL-13R $\alpha 2$ $\left(\mathrm{IL}-13 \mathrm{Ra2} 2^{--}\right)$were a gift from Michael J. Grusby (66) and were backcrossed for more than 10 generations onto a C57BL/6 background. Galectin-3-null mutant mice ( all $^{-/-}$) on a C57BL/6 background and wild-type C57BL/6J mice were purchased from The Jackson Laboratory. These mice were between 6 and 12 weeks old when used in these studies. All animals were humanely anesthetized with $100 \mathrm{mg} / \mathrm{kg}$ ketamine and $10 \mathrm{mg} / \mathrm{kg}$ xylazine per mouse before any intervention. The protocols used in these studies were evaluated and approved by the Institutional Animal Care and Use Committee at Brown University.

Western blot analysis. Protein lysates from macrophages and whole mouse lungs were prepared with RIPA lysis buffer (Thermo Fisher Scientific) containing protease inhibitor cocktail (Thermo Fisher Scientific) per the manufacturer's instructions. Twenty to thirty micrograms of lysate protein was subjected to electrophoresis on a $4 \%-15 \%$ gradient mini-Protean TGX gel (Bio-Rad). It was then transferred to a membrane using a semi-dry method with a Trans-Blot Turbo Trans- 
fer System (Bio-Rad). Membranes were blocked with Tris-buffered saline with Tween-20 (TBST) with 5\% nonfat milk for 1 hour at room temperature. After blocking, the membranes were incubated with the following primary antibodies overnight at $4^{\circ} \mathrm{C}$ in TBST and 5\% BSA: anti-mouse PD-L1 (10F.9G2, Bio X Cell), anti-mouse CHI3L1 (clone 321924, MAB2649, R\&D Systems), anti-human PD-L1 (MIH1, Thermo Fisher Scientific), and anti-human/mouse $\beta$-actin-HRP (4967, Cell Signaling Technology). The membranes were then washed 3 times with TBST and incubated with secondary antibodies in TBST with 5\% nonfat milk for 1 hour at room temperature. After 3 additional TBST washes, SuperSignal West Femto Maximum Sensitivity Substrate Solution (Thermo Fisher Scientific) was added to the membrane, and immunoreactive bands were detected using a ChemiDoc (Bio-Rad) imaging system.

RNA extraction and real-time quantitative PCR. Total cellular RNA was obtained using TRIzol reagent (Thermo Fisher Scientific) followed by RNA extraction using RNeasy Mini Kit (Qiagen) according to the manufacturer's instructions. mRNA was measured and used for real-time reverse transcriptase (RT) PCR as described previously (16, 20). The primer sequences used in these studies are summarized in Supplemental Table 1. Ct values of the test genes were normalized to the internal housekeeping gene $\beta$-actin.

Generation of anti-CHI3L1 and humanized bispecific antibodies against CHI3L1 and PD-1. The murine monoclonal anti-CHI3L1 antibody (FRG) was generated using peptide antigen (FRGQEDASPDRF, amino acids 223-234 of human CHI3L1) as its immunogen. This monoclonal antibody specifically detects both human and mouse CHI3L1 with high affinity $\left(K_{D} \approx 1.1 \times 10^{-9}\right)$. CHI3L1 (FRG) $\times$ PD-1 bispecific antibodies were generated with the Fc portion of FRG antibody (IgG1 isotype) in which anti-PD-1 was linked to FRG via its light chain (Supplemental Figure 8A). HEK293T cells were transfected with the bivalent FRGxPD-1 construct using Lipofectamine 3000 (L3000015, Invitrogen). Supernatant was collected for 7 days, and the antibody was purified using a protein A column (89960, Thermo Fisher Scientific). Ligand binding affinity and sensitivity were assessed using ELISA techniques. The affinities of this bispecific antibody to recombinant human CHI3L1 and PD-1 were both in the nanomolar ranges $\left(K_{D} \approx 1.1 \times 10^{-9}\right)$ by competitive ELISA (Supplemental Figure $\left.8 \mathrm{~B}\right)$. The original murine anti-PD-1 monoclonal antibody (67) was a gift from Lieping Chen (Medical Oncology, Yale School of Medicine). At the end of the preparation of the bispecific antibody, endotoxin levels were below the limits of detection of the Limulus amebocyte lysate assay.

Melanoma lung metastasis and antibody treatment. B16-F10, a mouse melanoma cell line, was purchased from ATCC (catalog CRL6475) and maintained in DMEM supplemented with 10\% FBS and $1 \%$ penicillin and streptomycin. When the cells formed an $80 \%$ confluent monolayer, they were collected, adjusted to the concentration of $10^{6}$ cells $/ \mathrm{mL}$, and injected into the mice via their lateral tail veins $\left(2 \times 10^{5}\right.$ cells per mouse in $200 \mu \mathrm{L}$ of DMEM). As previously described, i.p. injection of the noted doses of anti-CHI3L1 (FRG) and anti-PD-1, alone and in combination, and isotype control IgG (IgG2b) was started on the day of the B16 tumor cell challenge and continued every other day for 2 weeks $(42,43)$. Metastasis was assessed and quantified by counting of the melanoma colonies (black dots) on the pleural surface as previously described $(42,43)$.

Experiments with B16-BL6 cells. B16-BL6 cells were obtained from the Korean Cell Line Bank (no. 8006) and grown to confluence. They were then injected into the right hind footpad of C57BL/6 WT mice at a dose of $2 \times 10^{4}$ cells per mouse. Two weeks later the mice were randomized to FRG or its isotype control administered twice a week via an i.p. route. After an additional 3 weeks, footpads and popliteal lymph nodes were compared. The size (radius and surface area) of popliteal lymph nodes was assessed using the Motic Images (v3.0) analysis program (Motic Microscopes) and a stereomicroscope (Olympus SZX12).

FACS analysis. Single-cell suspensions from whole mouse lungs were prepared using the Lung Dissociation Kit (Miltenyi Biotec) per the manufacturer's instructions. Cells were stained with fluorescently labeled antibodies directed against CD45 (30-F11, 103126, Thermo Fisher Scientific), PD-L1 (MIH5, 12-5982-8, Thermo Fisher Scientific), PD-L2 (TY25, 12-5986-82, Thermo Fisher Scientific), CC10 (E-11, Santa Cruz Biotechnology), surfactant apoprotein C (SP-C; H-8, Santa Cruz Biotechnology), CD3-APC (145-2C1, MA1-10186, Thermo Fisher Scientific), CD68-PE (FA-11, Bio-Rad), CD11b-FITC (M1/70, 11-0112041, Thermo Fisher Scientific), and MHC-II (M5/114.15.2, 14-5321-82, Thermo Fisher Scientific). Flow cytometry data were collected using the BD FACSAria IIIu and analyzed with FlowJo (v10) software.

Immunohistochemistry. Formalin-fixed paraffin-embedded lung tissue blocks were serially sectioned at $5 \mu \mathrm{m}$ thickness and mounted on glass slides. After deparaffinization and dehydration, heat-induced epitope retrieval was performed by boiling of the samples in a steamer for 30 minutes in antigen unmasking solution (Antigen Retrieval Buffer, $100 \times$ citrate buffer, $\mathrm{pH}$ 6.0, Abcam). To prevent nonspecific protein binding, all sections were blocked in a ready-to-use serumfree protein blocking solution (Dako/Agilent) for 10 minutes at room temperature. The sections were then incubated with primary antibodies - anti-PD-L1 (10F.9G2, BE0101, InVivoMAb antibody, Bio X Cell), anti-CC10 (E-11, Santa Cruz Biotechnology), anti-SP-C (M-20, Santa Cruz Biotechnology), and anti-CD68 (ab125212, Abcam) - overnight at $4^{\circ} \mathrm{C}$. After 3 washings, fluorescence-labeled secondary antibodies were incubated for 1 hour at room temperature. The sections were then counterstained with DAPI, and coverslips were added.

Double-label immunohistochemistry. Double-label immunohistochemistry was used as previously described by our laboratory (21).

Macrophage preparation and treatment. Murine peritoneal and bone marrow-derived macrophages were prepared as previously described (68). They were then incubated with the noted concentrations of recombinant mouse CHI3L1 or IFN- $\gamma$ (R\&D Systems) or CAY10471 (Cayman Chemical). After incubating for the desired periods of time, the macrophages were collected and evaluated by RT-PCR, Western blotting, or flow cytometric assays as noted above.

$T$ cell culture and activation. TALL-104 T cells $\left(1 \times 10^{5}\right)$ were grown in complete RPMI (10\% FBS and 1\% penicillin and streptomycin). They were then activated by incubation with anti-human CD3 antibody (5 $\mu \mathrm{g} / \mathrm{mL}$; BioLegend) and anti-human CD28 antibody (5 $\mu \mathrm{g} /$ $\mathrm{mL}$; MABF408, Millipore) simultaneously for 2 hours in $5 \% \mathrm{CO}_{2}$ and air at $37^{\circ} \mathrm{C}$. After the incubation, the cells were washed twice to remove the extra antibody.

Melanoma cells and TALL-104 cell cocultures. Human melanoma A375 cell line (CRL-1619) and B16-F10 mouse melanoma cells (CRL-6475) were purchased from ATCC and cultured according to the instructions provided by the vendor. The TALL-104 cells $\left(1 \times 10^{5}\right)$ were cultured and activated as described above. After incubation, the cells were washed, and B16 melanoma and activated TALL-104 cells were resuspended together at a 1:6 ratio in complete RPMI media, dis- 
pensed into the multi-well slide chambers, and incubated in $5 \% \mathrm{CO}_{2}$ and air at $37^{\circ} \mathrm{C}$. One hour later, the cocultured cells were treated with the isotype control antibody or test antibodies. The effects of the isotype control antibodies were compared with the effects of antibodies against CHI3L1 (FRG; $5 \mu \mathrm{g}$ ), PD-1 (5 $\mu$ g), FRG plus PD-1 administered simultaneously (2.5 $\mu \mathrm{g}$ each), and FRGxPD-1 (5 $\mu \mathrm{g})$. After incubation for an additional 48 hours, cells were subjected to TUNEL evaluations and immunofluorescence evaluations of CD8, perforin, granzyme B, and PTEN as described below.

Measurement of cellular apoptosis and cytotoxic cell death responses. TUNEL staining using fluorescein-labeled dUTP was used to assess apoptosis and cytotoxic cell death responses. After 48-hour incubation with test and control antibodies, cocultured cells of prestained TALL104 (cyanin blue) and tumor cells (red) were fixed in $4 \%$ paraformaldehyde in PBS, permeabilized, and blocked. In between each step, the cells were washed twice with PBS (1x). The cells were then stained with the In Situ Cell Death Detection Kit, Fluorescein (Roche), per the manufacturer's instructions.

Immunofluorescence staining. To evaluate the activation and differentiation of cocultured T cells and expression of the tumor suppressor PTEN, immunofluorescence staining was carried out using antibodies against perforin (1:1000; B-D48, BioLegend), granzyme B (1:1000; GB11, BioLegend), CD8 (1:1000; HIT8a, BD Biosciences), and PTEN (1:1000; 9552, Cell Signaling Technology). After 48-hour incubation with test and control antibodies, cocultured cells were fixed in $4 \%$ paraformaldehyde in PBS, permeabilized, and blocked. Then the cells were incubated overnight at $4^{\circ} \mathrm{C}$ with primary antibodies noted above, washed twice with PBS (1×), and incubated for 2 hours at $37^{\circ} \mathrm{C}$ with secondary detection antibodies (1:500) and phalloidin (1:3000) (Invitrogen) for cytoskeleton staining. The cells were then washed, mounted with VECTASHIELD Antifade Mounting Medium (Vector Laboratories Inc.), and evaluated at $\times 20$ via fluorescence microscopy.

Statistics. Statistical evaluations were undertaken with GraphPad Prism software. As appropriate, groups were compared with 2-tailed Student's $t$ test or with nonparametric Mann-Whitney $U$ test. Values are expressed as mean \pm SEM. One-way ANOVA with Tukey's post hoc test was used for multiple-group comparisons. Statistical significance was defined as a level of $P$ less than 0.05 .

Study approval. The protocols used in these studies were evaluated and approved by the Institutional Animal Care and Use Committee at Brown University.

\section{Author contributions}

$\mathrm{BM}, \mathrm{JAE}, \mathrm{CGL}, \mathrm{SK}$, and BA conceived and designed the study. BM, BA, SK, and CML collected data. BM, BA, SK, CML, CHH, JSK, CGL, and JAE analyzed and interpreted data. JAE and CGL drafted the manuscript and revised it for important intellectual content.

\section{Acknowledgments}

This work was supported by NIH grants U01 HL108638 (to JAE), P01 HL114501 (to JAE), and R01 HL115813 (to CGL) from the National Heart, Lung, and Blood Institute; P20 GM103652 (to CML) from the National Institute of General Medical Sciences; and USAMRMC W81XWH-17-1-0196 (to JAE) from the US Department of Defense.

Address correspondence to: Jack A. Elias, Molecular Microbiology and Immunology Department, Brown University, 185 Meeting Street, Providence, Rhode Island 02912, USA. Email: jack_elias@ brown.edu.
1. Miller JF, Sadelain M. The journey from discoveries in fundamental immunology to cancer immunotherapy. Cancer Cell. 2015;27(4):439-449.

2. Topalian SL, et al. Immune checkpoint blockade: a common denominator approach to cancer therapy. Cancer Cell. 2015;27(4):450-461.

3. Chen J, et al. Regulation of PD-L1: a novel role of pro-survival signalling in cancer. Ann Oncol. 2016;27(3):409-416.

4. Nguyen LT, Ohashi PS. Clinical blockade of PD1 and LAG3 - potential mechanisms of action. Nat Rev Immunol. 2015;15(1):45-56.

5. Sun C, et al. Regulation and function of the PD-L1 checkpoint. Immunity. 2018;48(3):434-452.

6. Herbst RS, et al. Predictive correlates of response to the anti-PD-L1 antibody MPDL3280A in cancer patients. Nature. 2014;515(7528):563-567.

7. Goldberg SB, et al. Pembrolizumab for patients with melanoma or non-small-cell lung cancer and untreated brain metastases: early analysis of a non-randomised, open-label, phase 2 trial. Lancet Oncol. 2016;17(7):976-983.

8. Herbst RS, et al. Pembrolizumab versus docetaxel for previously treated, PD-L1-positive, advanced non-small-cell lung cancer (KEYNOTE-010): a randomised controlled trial. Lancet. 2016;387(10027):1540-1550.

9. Ritprajak P, Azuma M. Intrinsic and extrinsic control of expression of the immunoregulatory molecule PD-L1 in epithelial cells and squamous cell carcinoma. Oral Oncol. 2015;51(3):221-228.

10. Funkhouser JD, Aronson NN. Chitinase family GH18: evolutionary insights from the genomic history of a diverse protein family. BMC Evol Biol. 2007;7:96.

11. Aerts JM, et al. Biomarkers for lysosomal storage disorders: identification and application as exemplified by chitotriosidase in Gaucher disease. Acta Paediatr. 2008;97(457):7-14.

12. Morrison BW, Leder P. neu and ras initiate murine mammary tumors that share genetic markers generally absent in c-myc and int-2-initiated tumors. Oncogene. 1994;9(12):3417-3426.

13. Lee CG. Chitin, chitinases and chitinase-like proteins in allergic inflammation and tissue remodeling. Yonsei Med J. 2009;50(1):22-30.

14. Lee CG, et al. Role of chitin and chitinase/ chitinase-like proteins in inflammation, tissue remodeling, and injury. Annu Rev Physiol. 2011;73:479-501.

15. Lee CG, Elias JA. Role of breast regression protein-39/YKL-40 in asthma and allergic responses. Allergy Asthma Immunol Res. 2010;2(1):20-27.

16. Lee CG, et al. Role of breast regression protein 39 (BRP-39)/chitinase 3-like-1 in Th2 and IL-13induced tissue responses and apoptosis. J Exp Med. 2009;206(5):1149-1166.

17. Dela Cruz CS, et al. Chitinase 3-like-1 (CHI3L1) regulation of streptococcus pneumoniae lung infection. Cell Host Microbe. 2012;12(1):34-46.
18. $\mathrm{He} \mathrm{CH}$, et al. Chitinase 3-like 1 regulates cellular and tissue responses via IL-13 receptor $\alpha 2$. Cell Rep. 2013;4(4):830-841.

19. Kanneganti M, et al. Animal models of colitisassociated carcinogenesis. JBiomed Biotechnol. 2011;2011:342637.

20. Sohn MH, et al. The chitinase-like proteins breast regression protein-39 and YKL-40 regulate hyperoxia-induced acute lung injury. Am J Respir Crit Care Med. 2010;182(7):918-928.

21. Lee CM, et al. IL-13Ralpha2 uses TMEM219 in chitinase 3-like-1-induced signalling and effector responses. Nat Commun. 2016;7:12752.

22. Libreros S, Iragavarapu-Charyulu V. YKL-40/ CHI3L1 drives inflammation on the road of tumor progression. JLeukoc Biol. 2015;98(6):931-936.

23. Zhou Y, et al. Chitinase 3-like-1 and its receptors in Hermansky-Pudlak syndrome-associated lung disease. J Clin Invest. 2015;125(8):3178-3192.

24. Matsuura $\mathrm{H}$, et al. Role of breast regression protein-39 in the pathogenesis of cigarette smoke-induced inflammation and emphysema. Am J Respir Cell Mol Biol. 2011;44(6):777-786.

25. Ober C, et al. Effect of variation in CHI3L1 on serum YKL-40 level, risk of asthma, and lung function. NEngl JMed. 2008;358(16):1682-1691.

26. Chen CC, et al. Chitinase 3-like-1 expression in colonic epithelial cells as a potentially novel marker for colitis-associated neoplasia. Am J Pathol.2011;179(3):1494-1503. 
27. Choi IK, et al. High serum YKL-40 is a poor prognostic marker in patients with advanced non-small cell lung cancer. Acta Oncol. 2010;49(6):861-864.

28. Coffman FD. Chitinase 3-Like-1 (CHI3L1): a putative disease marker at the interface of proteomics and glycomics. Crit Rev Clin Lab Sci. 2008;45(6):531-562.

29. Hottinger AF, et al. YKL-40 and MMP-9 as serum markers for patients with primary central nervous system lymphoma. Ann Neurol. 2011;70(1):163-169.

30. Iwamoto FM, et al. Serum YKL-40 is a marker of prognosis and disease status in high-grade gliomas. Neuro Oncol. 2011;13(11):1244-1251.

31. Johansen JS, et al. Serum YKL-40: a new potential marker of prognosis and location of metastases of patients with recurrent breast cancer. Eur J Cancer. 1995;31A(9):1437-1442.

32. Johansen JS, et al. Serum YKL-40, a new prognostic biomarker in cancer patients? Cancer Epidemiol Biomarkers Prev. 2006;15(2):194-202.

33. Johansen JS, et al. Plasma YKL-40: a potential new cancer biomarker? Future Oncol. 2009;5(7):1065-1082.

34. Peng C, et al. YKL-40 protein levels and clinical outcome of human endometrial cancer. J Int Med Res. 2010;38(4):1448-1457.

35. Schmidt H, et al. Elevated serum level of YKL40 is an independent prognostic factor for poor survival in patients with metastatic melanoma. Cancer. 2006;106(5):1130-1139.

36. Schmidt H, et al. Serum YKL- 40 predicts relapsefree and overall survival in patients with American Joint Committee on Cancer stage I and II melanoma. J Clin Oncol. 2006;24(5):798-804.

37. Shao R, et al. Breast cancer expression of YKL-40 correlates with tumour grade, poor differentiation, and other cancer markers. Br J Cancer. 2011;105(8):1203-1209.

38. Shao R, et al. YKL-40, a secreted glycoprotein, promotes tumor angiogenesis. Oncogene. 2009;28(50):4456-4468.

39. Thöm I, et al. Elevated pretreatment serum concentration of YKL-40-An independent prognostic biomarker for poor survival in patients with metastatic nonsmall cell lung cancer. Cancer.
2010;116(17):4114-4121.

40. Junker N, et al. Expression of YKL-40 by peritumoral macrophages in human small cell lung cancer. Lung Cancer. 2005;48(2):223-231.

41. Johansen JS, et al. High serum YKL-40 level in patients with small cell lung cancer is related to early death. Lung Cancer. 2004;46(3):333-340.

42. Ma B, et al. Role of chitinase 3-like-1 and semaphorin 7a in pulmonary melanoma metastasis. Cancer Res. 2015;75(3):487-496.

43. Ma B, et al. RIG-like helicase regulation of chitinase 3-like 1 axis and pulmonary metastasis. Sci Rep. 2016;6:26299.

44. Heidegger S, et al. RIG-I activation is critical for responsiveness to checkpoint blockade. Sci Immunol. 2019;4(39):eaau8943.

45. Garcia-Diaz A, et al. Interferon receptor signaling pathways regulating PD-L1 and PD-L2 expression. Cell Rep. 2017;19(6):1189-1201.

46. Mandai M, et al. Dual faces of IFN $\gamma$ in cancer progression: a role of $\mathrm{PD}-\mathrm{L} 1$ induction in the determination of pro- and antitumor immunity. Clin Cancer Res. 2016;22(10):2329-2334.

47. Zhou Y, et al. Galectin-3 interacts with the CHI3L1 axis and contributes to Hermansky-Pudlak syndrome lung disease. J Immunol. 2018;200(6):2140-2153.

48. Wolchok JD, et al. Overall survival with combined nivolumab and ipilimumab in advanced melanoma. N Engl J Med. 2017;377(14):1345-1356.

49. Hellmann MD, et al. Nivolumab plus ipilimumab in lung cancer with a high tumor mutational burden. N Engl J Med. 2018;378(22):2093-2104.

50. Motzer RJ, et al. Nivolumab plus ipilimumab versus sunitinib in advanced renal-cell carcinoma. NEngl JMed. 2018;378(14):1277-1290.

51. Dahlen E, et al. Bispecific antibodies in cancer immunotherapy. Ther Adv Vaccines Immunother. 2018;6(1):3-17.

52. Topalian SL. Targeting immune checkpoints in cancer therapy. JAMA. 2017;318(17):1647-1648.

53. Cohen N, et al. Fibroblasts drive an immunosuppressive and growth-promoting microenvironment in breast cancer via secretion of Chitinase 3-like 1. Oncogene. 2017;36(31):4457-4468.

54 . Hodi FS, et al. Improved survival with ipilimumab in patients with metastatic melanoma. $N$ Engl Med. 2010;363(8):711-723.

55. Lu D, et al. Beyond T cells: understanding the role of PD-1/PD-L1 in tumor-associated macrophages. JImmunol Res. 2019;2019:1919082.

56. Zhong X, et al. The role of tumor-associated macrophages in colorectal carcinoma progression. Cell Physiol Biochem. 2018;45(1):356-365.

57. Sato M, et al. Impaired production of Th1 cytokines and increased frequency of Th2 subsets in PBMC from advanced cancer patients. Anticancer Res. 1998;18(5d):3951-3955.

58. Geng B, et al. Chitinase 3-like 1-CD44 interaction promotes metastasis and epithelial-to-mesenchymal transition through $\beta$-catenin/Erk/Akt signaling in gastric cancer. J Exp Clin Cancer Res. 2018;37(1):208.

59. Okazaki T, Honjo T. The PD-1-PD-L pathway in immunological tolerance. Trends Immunol. 2006;27(4):195-201.

60. Siegel RL, et al. Cancer statistics, 2017. CA Cancer JClin. 2017;67(1):7-30.

61. Santini FC, Hellmann MD. PD-1/PD-L1 axis in lung cancer. Cancer J. 2018;24(1):15-19.

62. Yang Y. Cancer immunotherapy: harnessing the immune system to battle cancer. JClin Invest. 2015;125(9):3335-3337.

63. Rotte A. Combination of CTLA-4 and PD-1 blockers for treatment of cancer. J Exp Clin Cancer Res. 2019;38(1):255.

64. Pfirschke C, et al. Immunogenic chemotherapy sensitizes tumors to checkpoint blockade therapy. Immunity. 2016;44(2):343-354.

65. Yajima I, et al. RAS/RAF/MEK/ERK and PI3K/ PTEN/AKT signaling in malignant melanoma progression and therapy. Dermatol Res Pract. 2012;2012:354191.

66. Wood N, et al. Enhanced interleukin (IL)-13 responses in mice lacking IL-13 receptor alpha 2. JExp Med. 2003;197(6):703-709.

67. Dong H, et al. Tumor-associated B7-H1 promotes T-cell apoptosis: a potential mechanism of immune evasion. Nat Med. 2002;8(8):793-800.

68. Zhang $\mathrm{X}$, et al. The isolation and characterization of murine macrophages. Curr Protoc Immunol. 2008; Chapter 14:Unit 14.1. 